\title{
Mindfulness-based programs for substance use disorders: a systematic review of manualized treatments
}

\author{
J. Richard Korecki ${ }^{1}$, Frank J. Schwebel ${ }^{2}$, Victoria R. Votaw ${ }^{1,2}$ and Katie Witkiewitz ${ }^{1,2^{*}}$
}

\begin{abstract}
Background: Substance use disorders are prevalent and returning to substance use (i.e., relapse) following treatment is common, underscoring the need for effective treatments that will help individuals maintain long-term reductions in substance use. Mindfulness-based interventions (MBIs) have been increasingly developed and evaluated for the treatment of substance use disorders. The aim of this article was to update a systematic review conducted by Li et al. in 2017 on the outcomes of randomized control trials of MBIs for substance use disorders. In addition, we provided a session-by-session examination of the most widely used MBI protocols.

Methods: We conducted a comprehensive literature search of the PubMed, PsycINFO, and Web of Science databases from January of 2016 through April of 2020. Studies were included based on the following criteria: 1) examined the effects of an $\mathrm{MBI}, 2$ ) employed a randomized controlled trial design with repeated measures, including secondary data analyses of randomized controlled trials, and 3) enrolled participants seeking treatment for substance use disorders.
\end{abstract}

Results: The search identified 902 publications and 30 studies were eligible for inclusion and data extraction. MBls appear to be as effective as existing evidence-based treatments for substance use disorders at reducing the frequency and quantity of alcohol and drug use, substance-related problems, craving for substance use, and at increasing the rate of abstinence.

Conclusions: Future directions include additional large scale randomized controlled trials, investigation of the most suitable settings and protocols, examination of patient populations that may benefit most from MBIs, and dissemination and implementation research.

Keywords: Mindfulness, Mindfulness-based interventions, Addictive behavior, Behavior change, Substance use disorders, Treatment

\section{Introduction}

Substance use disorder (SUD) is a significant public health problem associated with considerable social and economic costs in the United States (U.S.) and throughout the world. According to the 2018 National Survey

\footnotetext{
* Correspondence: katiew@unm.edu

'Department of Psychology, University of New Mexico, 2650 Yale Blvd SE, Ste. 200, Albuquerque, NM 87106, USA

${ }^{2}$ Center on Alcohol, Substance use, And Addictions (CASAA), University of New Mexico, Albuquerque, USA
}

on Drug Use and Mental Health, approximately 20.3 million people ages 12 or older suffered from a SUD [1]. The estimated cost associated with substance use in the U.S. as measured by crime, lost work productivity, and health care is nearly $\$ 740$ billion annually [2]. Furthermore, approximately $40-60 \%$ of individuals relapse within the first year following SUD treatment [3]. The large-scale prevalence of SUDs and the frequency of relapse after SUD treatment underscores the need to

(c) The Author(s). 2020 Open Access This article is licensed under a Creative Commons Attribution 4.0 International License, which permits use, sharing, adaptation, distribution and reproduction in any medium or format, as long as you give appropriate credit to the original author(s) and the source, provide a link to the Creative Commons licence, and indicate if changes were made. The images or other third party material in this article are included in the article's Creative Commons licence, unless indicated otherwise in a credit line to the material. If material is not included in the article's Creative Commons licence and your intended use is not permitted by statutory regulation or exceeds the permitted use, you will need to obtain permission directly from the copyright holder. To view a copy of this licence, visit http://creativecommons.org/licenses/by/4.0/ The Creative Commons Public Domain Dedication waiver (http://creativecommons.org/publicdomain/zero/1.0/) applies to the data made available in this article, unless otherwise stated in a credit line to the data. 
develop effective treatments that will help individuals maintain long-term changes in substance use.

A treatment option for promoting long-term health behavior change that has gained popularity in recent years is mindfulness-based intervention (MBI). One definition of mindfulness includes paying attention in the present moment in a particular way: on purpose and without judgment [4]. It is experiencing the true nature of the moment at hand without the biases created from past experiences, or the expectations of future events. The practice of mindfulness meditation has been performed for thousands of years and was traditionally taught to reduce the suffering of the human experience and to cultivate well-being [5]. Over the last few decades, secularized meditation practices have been integrated into several more traditional Western healthcare settings.

Mindfulness-Based Stress Reduction (MBSR), developed in the early 1980s, was the first mindfulness-based practice introduced into Western health care settings [6, 7]. MBSR was initially developed for patients with chronic pain and early trials identified reductions in measures of pain, negative body image, mood disturbance, anxiety, and depression following MBSR [6-8]. Since the success of MBSR in the early 1980s, there have been several MBIs developed to treat a multitude of conditions as both standalone treatments and adjuncts to already established treatments. Such interventions include Mindfulness-Based Cognitive Therapy (MBCT) [9], Mindfulness-Based Eating, Mindfulness-Based Childbirth and Parenting [10], and other third-wave therapies that include aspects of mindfulness, such as Dialectical Behavior Therapy (DBT) [11] and Acceptance and Commitment Therapy (ACT) [12].

Several MBIs specifically designed for SUD have been developed such as Mindfulness-Based Relapse Prevention (MBRP) [13, 14], Mindfulness Oriented Recovery Enhancement (MORE) [15], Mindfulness Training for Smokers (MTS) [16], Moment-by-Moment in Women's Recovery (MMWR) [17], and other interventions designed to address factors theorized to maintain SUD. Craving is one such factor that is theorized to maintain SUD and targeted by MBIs. One of the major facets of MBIs for SUD is a non-judgmental observation of thoughts and behavioral urges. By divorcing oneself from the feelings associated with the craving (i.e. desire, aversion, physical or emotional discomfort, etc.), an individual may be able to "ride out" cravings without engaging in substance use. Another common aspect of addictive behaviors targeted by MBIs is the automaticity of action (i.e. "acting on autopilot" [18]). For example, MBIs target substance use as an automatic reaction when exposed to feelings, locations, and people that serve as cues for substance use. Mitigating automatic behaviors by bringing purposeful attention to the present moment may help to reduce the frequency and quantity of substance use and substance-related problems.

In recent years, several reviews and meta-analyses have investigated the efficacy of MBIs for the treatment of SUD. Li and colleagues [19] conducted a systematic review and meta-analysis of 42 studies that examined the effects of various MBIs on substance use. Findings from this review indicated that MBIs were more effective than control conditions (e.g., treatment as usual (TAU), relapse prevention treatment (RP), cognitive behavioral therapy (CBT), support group) at reducing the frequency and amount of substance use, the number of substancerelated problems, level of craving for substance use, and at increasing rates of abstinence [19]. A 2018 metaanalysis of MBIs for SUD conducted by Goldberg and colleagues [20] found that MBIs were generally equivalent to evidence-based conditions and superior to other control conditions (e.g., minimal treatment, non-specific active controls). However, the efficacy of MBIs, as compared to control conditions, differed by follow-up period (i.e., post-treatment versus longer-term follow-ups) and targeted disorder. These mixed results highlight the need for larger, randomized clinical trials and an understanding of the subgroups of participants that may respond best to MBIs. With the emergence of varied MBIs for SUD, there is a need for more research into the most effective duration, meditation techniques taught, settings, and potential participants for these mindfulness-based programs.

The purpose of this manuscript was twofold. First, we reviewed treatment protocols of MBIs for SUD. Second, we conducted a systematic review of randomized controlled trials (RCTs) of MBIs for SUD, with a focus on studies published after 2017 to update the systematic review conducted by Li et al. Specifically, we aimed to provide a detailed account (session-by-session outline) of the most frequently studied MBIs, followed by a description of the current outcomes of those interventions based on findings of the systematic review. We then synthesized information gathered from the systematic review to address clinical implications, current limitations in the field, and suggestions for the road ahead.

\section{Method}

\section{Search strategy}

Given our aim of updating and extending the $\mathrm{Li}$ et al. systematic review published in 2017, our search strategy and guidelines for study eligibility were guided by this prior manuscript. We searched PubMed, PsycINFO, and Web of Science databases from January of 2016 through April of 2020. Search terms included the following combinations: ('mindfulness' OR 'mindfulness intervention' OR 'mindfulness meditation' OR 'mindfulness treatment' 
OR 'mindfulness-based relapse prevention' OR 'mindfulness-based stress reduction') AND ('substance use' OR 'alcohol' OR 'cocaine' OR 'opioid' OR 'tobacco' OR 'marijuana' OR 'drug'). We also reviewed the reference list of eligible articles to identify additional studies not identified in our initial search. All methods were carried out in accordance with the Preferred Reporting Items for Systematic Reviews and Meta-Analyses (PRISMA) guidelines [21]. This systematic review was not preregistered prior to publication.

\section{Study selection and data extraction}

Peer-reviewed manuscripts published from January of 2016 to April of 2020 were included based on the following criteria: 1) examined the effects of an MBI, 2) employed an RCT design with repeated measures, including secondary data analyses of RCTs, and 3) enrolled participants seeking treatment for SUD. Studies were excluded for the following reasons: 1) non-peer reviewed publications, case reports, case series, editorials, commentaries, letters to the editor, book chapters, previously published narrative reviews, theses/dissertations, and study protocols, 2) only reported qualitative results, 3) non-randomized trials without a control group, 4) examined very brief mindfulness interventions (due to a lack of structured, manualized protocols and intervention content that varied from study to study), 5) did not assess substance use outcomes, 6) examined interventions without a formal mindfulness component (e.g., Acceptance and Commitment Therapy, Dialectic Behavior Therapy, etc.) or without a specific focus on SUD (e.g., Vipassana).

The first author (JRK) conducted the initial search. Authors JRK, FJS, and VRV determined eligibility, addressed eligibility questions, and performed data extraction. Data extraction was performed using a Microsoft Excel template to collect the following information (if available): citation (i.e., first author, year, journal); study aims; total sample size and sample size in the experimental and control conditions; overview of the population (e.g., demographics, relevant recruitment methods); description of the treatment and control conditions; targeted behavior; duration of treatment and timing of follow-up assessments; treatment compliance and attrition; additional notes on methodology; overview of results; and notes/limitations.

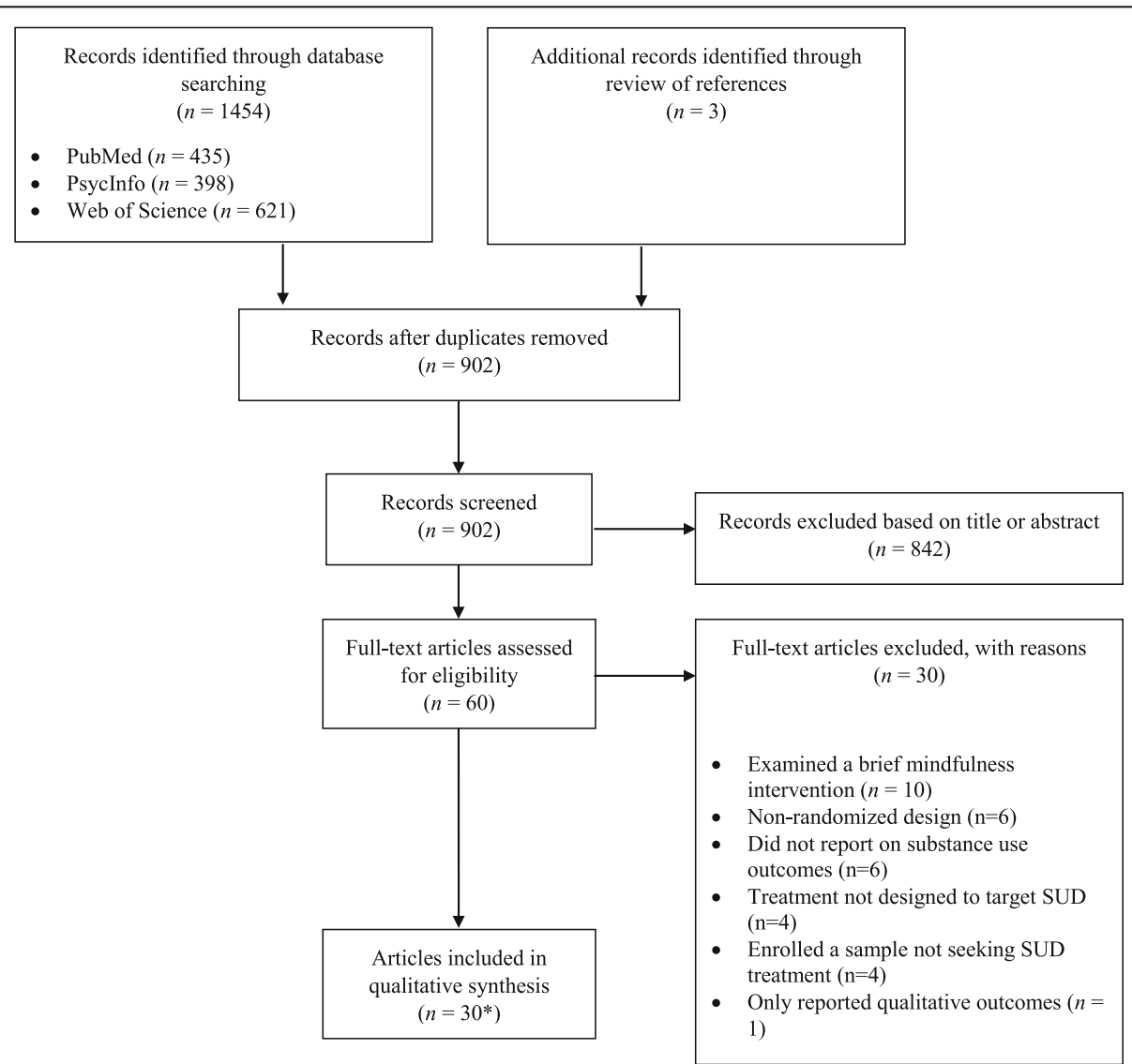

Fig. 1 Flow diagram of records identified, screened, and included. Note: Records could be excluded for multiple reasons. *Includes one relevant erratum of an eligible manuscript 


\section{Results}

Results of the search are presented in Fig. 1. Our search identified 902 publications, of which, 60 full-text articles were assessed for eligibility. Thirty of the 60 articles were excluded for the following reasons: examined a brief mindfulness intervention $(n=10)$, employed a nonrandomized design $(n=6)$, did not report on substance use outcomes $(n=6)$, active treatment not designed to target SUD $(n=4)$, enrolled a sample not seeking SUD treatment $(n=4)$, and only reported qualitative outcomes $(n=1)$; articles could be excluded for multiple reasons. Overall, 30 studies and one relevant erratum of an eligible study were eligible for inclusion and data extraction. A session-by-session outline of themes, primary practices, mechanisms studied, and moderators investigated for each of the six manualized protocols is presented in Table 1. Characteristics of included studies are presented in Table 2 .

\section{Mindfulness-based relapse prevention (MBRP) Overview of protocol}

MBRP is a manualized, structured protocol that integrates formal meditation practices with the cognitive behavioral approach of relapse prevention treatment. The core goals of MBRP are to: 1) cultivate awareness of internal (e.g., emotions, thoughts) and external (e.g., environmental) substance use cues to create opportunities to address triggers rather than instinctively using substances; and 2) practice (both via imagination and in vivo exposure) remaining with unpleasant affective, cognitive, or physical experiences without automatically seeking to escape or avoid the situation [13, 54]. MBRP courses are typically in a group format and consist of eight, 120-min sessions. Each session begins with a guided mindfulness practice and is followed by "inquiry," a discussion (similar to Socratic questioning) about the client's present moment experience of the practice. The first session uses experiential exercises focused on bodily sensations (e.g., taste and smell) designed to introduce the rationale for mindfulness by examining the role that "autopilot" plays in daily life and contrasting it with mindful awareness of experiences. The second and third sessions shift to other aspects of current experience such as sight and sound. Clients are encouraged to notice triggers and urges for substance use and to practice exercises throughout the day to encourage exiting autopilot and increasing mindfulness. Sessions four through six focus on mindfulness of thoughts and emotions. For example, clients begin to bring awareness to patterns of behavior and antecedents to relapse and are taught to notice thoughts, sensations, or emotions that might arise while remaining focused on the present moment experience. Clients are instructed to remain mindful of the present moment despite the unpleasant sensation as a method of allowing time and space to make a less impulsive, skillful decision. In sessions seven and eight, clients discuss generalizing learned practices and facilitating an environment that can support continued practice of mindfulness and changes made to substance use.

MBRP is one of, if not the most, researched MBI for SUD. Bowen and colleagues [55] conducted an initial pilot feasibility and efficacy trial of MBRP. They found MBRP to be feasible to implement and found initial support for efficacy as measured by significantly lower substance use rate, decreases in craving, and increases in acceptance and acting with awareness as compared to TAU. Other large scale RCTs and meta-analyses have shown the efficacy of MBRP with a range of samples (e.g., [56, 57]), treatment delivery modalities (e.g., [13, $56,57])$, and treatment targets $[14,56,58]$. Recent research includes RCTs designed to replicate or expand earlier findings (i.e., to new populations, examining adjunctive interventions) and secondary data analyses that seek to identify potential mechanisms of change and treatment moderators.

\section{Literature review}

Expansion of MBRP findings: specific populations, modified protocols, and adjunctive interventions $\mathrm{Sev}$ eral recent studies have aimed to expand previous findings on MBRP to specific populations with SUD. For example, Glasner-Edwards and colleagues compared MBRP to health education (control condition) among stimulant dependent adults (DSM-IV criteria) receiving a 12-week, 24 session, contingency management intervention $(n=63)$ [35]. MBRP sessions were shortened to $75 \mathrm{~min}$ to increase engagement with this population. There were no significant differences between MBRP and control on negative urinalysis rate or Addiction Severity Index score at post-treatment and one-month follow-up. However, MBRP was associated with lower odds of stimulant use among individuals with depressive and anxiety disorders. Participants in the MBRP condition reported greater reductions in depressive symptoms (Cohen's $d=0.58$ ) and psychiatric severity (Cohen's $d=$ 0.61 ) over time relative to control.

Modifications of MBRP for specific substances have also recently been evaluated. In an RCT comparing the efficacy of MBRP for alcohol use disorder (adapted to focus on alcohol-specific content) relative to TAU among adults in outpatient substance use treatment, Zgierska and colleagues did not find significant posttreatment differences on any of the constructs of interest (alcohol consumption, drinking-related consequences, perceived stress, or mindfulness) [45]. Weiss de Souza and colleagues compared the effects of MBRP in 
王辛王

일

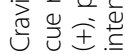

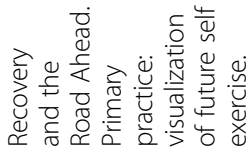

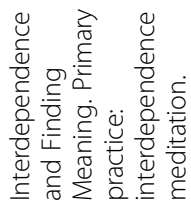

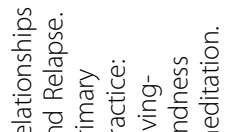

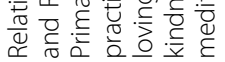

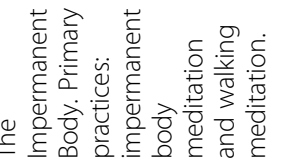

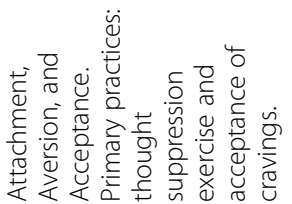

它它紊

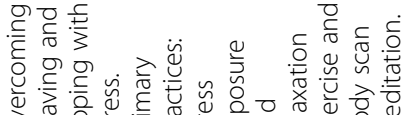

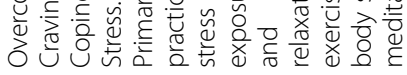

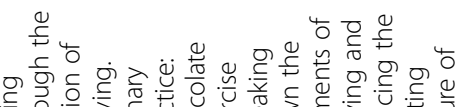

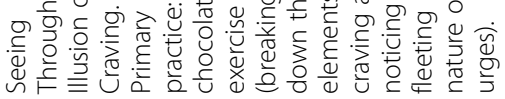

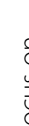
के

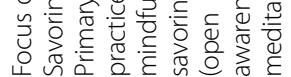

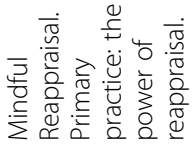

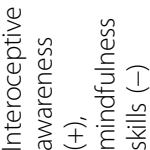

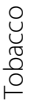

$\frac{\$}{z}$

$\frac{\$}{z}$

$\stackrel{\varangle}{z}$

$\frac{\pi}{z}$

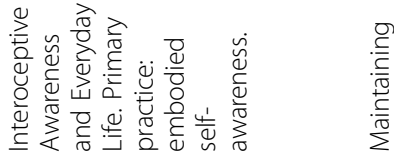

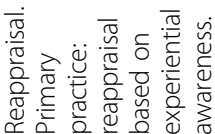

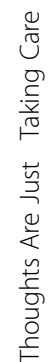

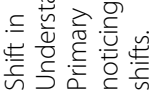

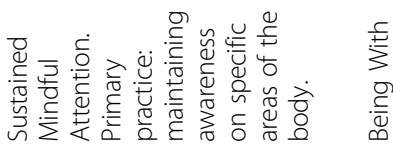

立

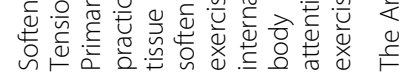

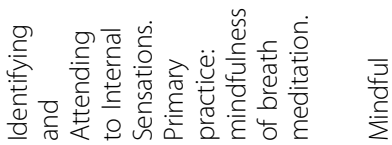

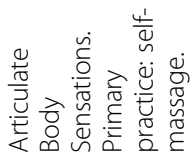

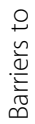
岕

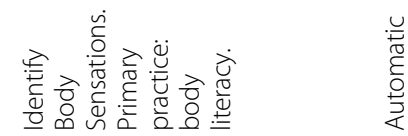

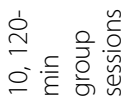

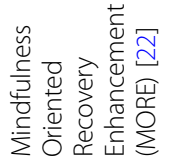

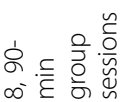

$\underset{\substack{\grave{1} \\ \infty}}{\infty}$

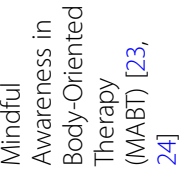

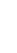

总

政

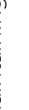




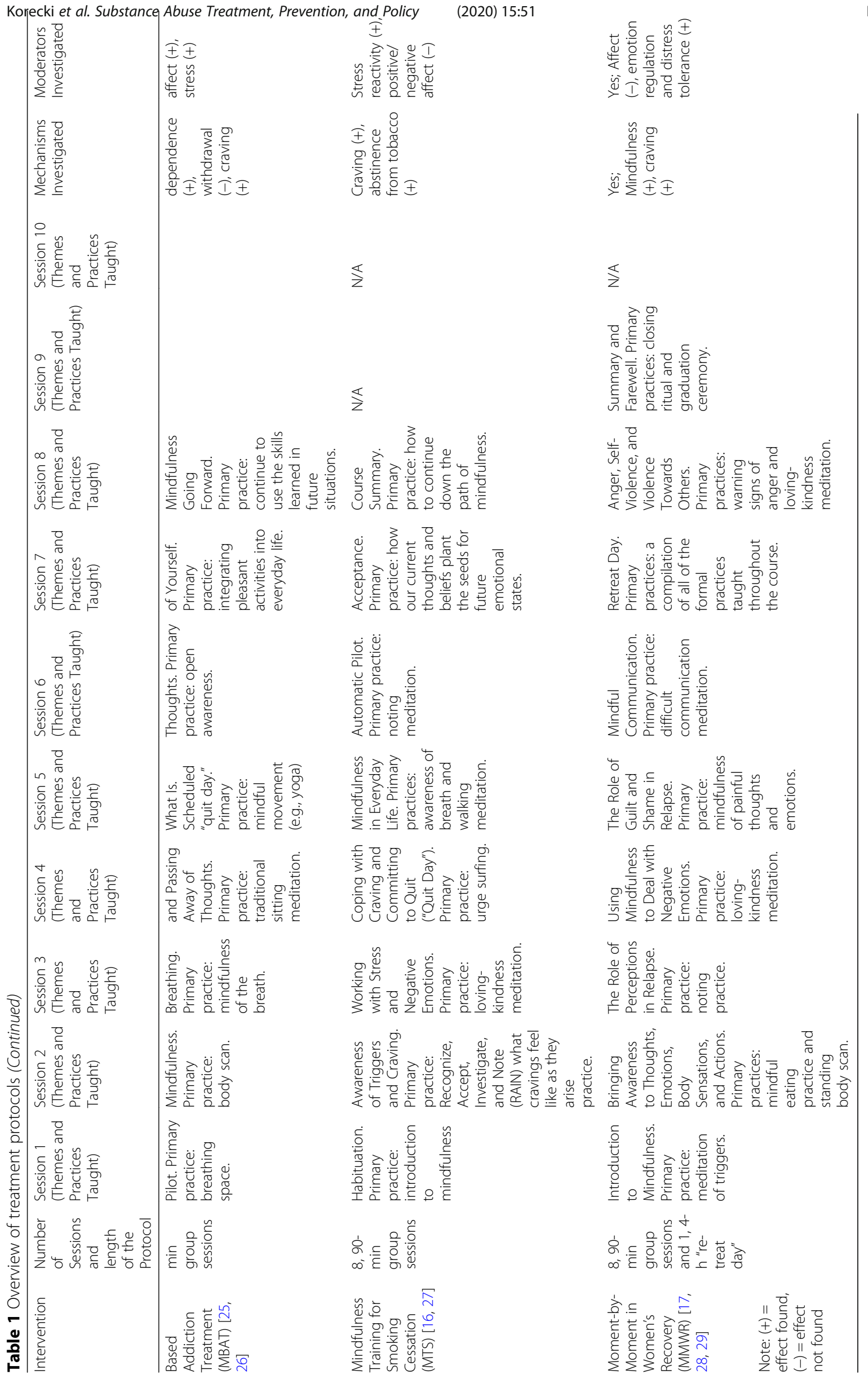


Table 2 Characteristics of Studies Included in Systematic Review

\begin{tabular}{|c|c|c|c|c|}
\hline Reference & $\begin{array}{l}\text { Treatment } \\
\text { condition }\end{array}$ & $\begin{array}{l}\text { Control } \\
\text { condition(s) }\end{array}$ & Overview of sample & $\begin{array}{l}\text { Data collection } \\
\text { time points }\end{array}$ \\
\hline \multicolumn{5}{|c|}{ Mindfulness-Based Relapse Prevention (MBRP) } \\
\hline $\begin{array}{l}\text { Abed } \\
\text { et al., } 2019 \\
\text { [30] }\end{array}$ & $\begin{array}{l}\text { MBRP + } \\
\text { Methadone } \\
\text { Maintenance } \\
\text { Therapy (MMT): } \\
\text { 8, 120-min } \\
\text { sessions. }\end{array}$ & $\begin{array}{l}\text { No intervention } \\
\text { control + MMT. }\end{array}$ & $\begin{array}{l}60 \text { adult Iranian } \\
\text { males (MRBP - } 30 \\
\text { participants ( } 26 \\
\text { completed), Control } \\
-30 \text { participants ( } 29 \\
\text { completed)) } \\
\text { undergoing MMT in } \\
\text { Isfahan, Iran. } \\
\text { Participants were } \\
\text { between the ages } \\
\text { of } 27 \text { and } 50 \text { who } \\
\text { had undergone } \\
\text { MMT within the six } \\
\text { months to one year } \\
\text { prior to the } \\
\text { beginning of the } \\
\text { study. Prior heroin } \\
\text { use ranged from } \\
\text { two to seven years. }\end{array}$ & $\begin{array}{l}\text { Baseline and } \\
\text { post- } \\
\text { intervention } \\
\text { testing of the } \\
\text { Heroin Craving } \\
\text { Questionnaire } \\
\text { (HCQ), and a } \\
\text { urinalysis the } \\
\text { first, second, } \\
\text { and third } \\
\text { months after } \\
\text { the eight-week } \\
\text { intervention. }\end{array}$ \\
\hline
\end{tabular}

Brown Rolling MBRP + Rolling MBRP + et al., 2020 tDCS: 8, 120- sham tDCS: 8,

[31] min sessions 120-min sessions with the first with the first 3030-min consist- min consisting of ing of tDCS. the sham $\mathrm{tDCS}$.
68 adults interested Baseline and in reducing their drinking (Active $\mathrm{tDCS}=36$; sham tDCS $=32) .52 .9 \%$ male, 52.16 (13.6) years old, $50.0 \%$ non-Hispanic white. 98.5\% met DSM-5 criteria for current AUD with the remaining participant meeting criteria for lifetime
Primary outcomes The mean of were differences the post-test among the scores of scores in the craving and desire subscales of de(HCQ) and lapse occurrence (drug urine tests). sire to use on the HCQ (pretest $M=34.27$; post-test $M=$ 18.93), intention to use (pre-test $M=30.66$; post test $M=18.42$ ) and anticipation of relief from withdrawal or dysphoria (pretest $M=38.58$; post-test $M=$

21.66) decreased from pre-test to post-test in the MBRP group.

The results revealed a significant difference among the scores in the subscales of craving and the difference in the three subscales of desire to use, intention to use, and anticipation of relief from withdrawal or dysphoria was statistically significant. There was a marked reduction in positive drug tests for the MBRP group ( 9 ,

7, 14\%) compared with the control $(23,24$

22\%) over the three-month follow-up window.

Alcohol cue-related hypersensitivity: EEG was recorded to capture an eventrelated component shown to relate to emotionally salient stimuli. The late positive potential (LPP), and self-reported

There was a main effect of time in predicting craving ratings and LPP amplitudes for the alcohol images, such that craving craving and negative ratings and LPP affect were recorded amplitudes during an image significantly presentation task. decreased over
There is a potential lack of generalizability in the study. The population was comprised of only male MMT patients. Without an active control group, it is hard to determine the effects of MBRP vs. the MMT.
The retention for the EEG assessment from the baseline to the post- treatment follow-up was low $54.4 \%$ completed both assessments). 
Table 2 Characteristics of Studies Included in Systematic Review (Continued)

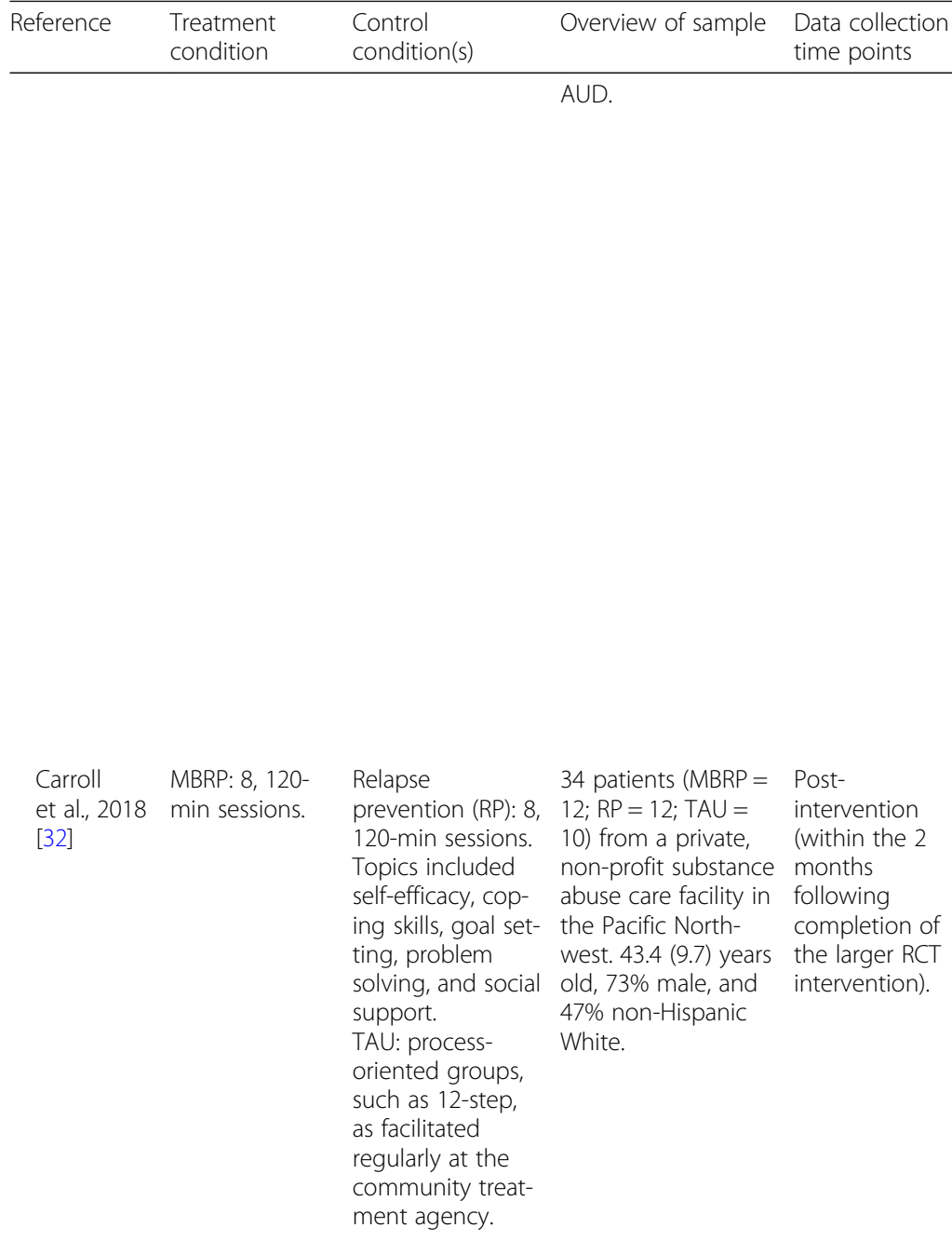

$\begin{array}{llll}\text { Davis et } & \text { Rolling MBRP + } & \text { TAU + treatment } & 79 \text { young adults } \\ \text { at., } 2018 & \text { treatment } & \text { normally } & \text { Baseline, bi- } \\ \text { [33] } & \text { normally } & \text { provided by the } & \text { 35) enrolled in a }\end{array} \quad \begin{aligned} & \text { weekly (every } \\ & 2 \text { weeks) as- }\end{aligned}$

[33] normally provided by provided by the
residential facility:
2 weeks) assessments
The primary

outcome was tonic and phasic heart rate variability (HRV) to a cognitive stressor

(Electrocardiography (ECG)). Secondary outcomes included self- reported anxiety and craving during the cognitive stressor.

Primary outcomes were perceived stress (PSS), craving (GAIN assessment),
Results

Limitations

time Also, there

was an effect of

group

attendance,

such that more

groups

attended was

associated with

lower craving

rations. There

was no effect of

active versus

sham tDCS in

predicting

craving ratings.

Significantly

higher LPP

amplitudes

were associated

with the active

tDCS compared

with the sham

tDCS.

Prior to

performing the stressor task,

MBRP evinced

greater tonic

HF-HRV than RP,

HF-HRV did not

significantly dif-

fer between RP

and TAU. All of

the pairwise

comparisons

were significant

(TAU vs RP, $p=$

0.013; TAU vs

MBRP, $p<0.001$;

RP vs MBRP, $p=$

0.011 ) for phasic

HRV. State anx-

iety increased

during the stres-

sor and quickly

subsided during

recovery in all

three treatment

groups. Groups

did not differ

significantly in

baseline or re-

covery values,

nor did they dif-

fer in rates of re-

turn to baseline.

Group differ-

ences in base-

line, recovery, or rates of recovery

for craving were not observed.

Those assigned Small sample to the TAU size, but this is group tended the first study to show
This laboratory assessment was conducted as an extension of a largecale clinical trial, and in consideration of participant burden, there is only one assessment time point with a relatively small sample. 
Table 2 Characteristics of Studies Included in Systematic Review (Continued)

\begin{tabular}{|c|c|c|c|c|c|c|c|}
\hline Reference & $\begin{array}{l}\text { Treatment } \\
\text { condition }\end{array}$ & $\begin{array}{l}\text { Control } \\
\text { condition(s) }\end{array}$ & Overview of sample & $\begin{array}{l}\text { Data collection } \\
\text { time points }\end{array}$ & Outcome measures & Results & Limitations \\
\hline & $\begin{array}{l}\text { the residential } \\
\text { facility: } 8,90- \\
\text { min classes } \\
\text { that were held } \\
\text { twice weekly. } \\
\text { Members were } \\
\text { enrolled as } \\
\text { they entered } \\
\text { the residential } \\
\text { facility (as } \\
\text { opposed to the } \\
\text { standard 8- } \\
\text { week cohort- } \\
\text { based proto- } \\
\text { col). The basic } \\
\text { treatment prac- } \\
\text { tice employed } \\
\text { at the residen- } \\
\text { tial treatment } \\
\text { center was a } \\
\text { mix of } \\
\text { cognitive- } \\
\text { behavioral } \\
\text { treatment and } \\
12-\text { step ap- } \\
\text { proach to } \\
\text { recovery. }\end{array}$ & $\begin{array}{l}\text { 8, 90-min sessions } \\
\text { held twice weekly } \\
\text { that were social } \\
\text { support groups } \\
\text { (Alcoholics and } \\
\text { Narcotics } \\
\text { Anonymous). }\end{array}$ & $\begin{array}{l}\text { treatment facility. } \\
25.3 \text { ( } 2.7) \text { years old, } \\
\text { the majority of } \\
\text { clients were non- } \\
\text { Hispanic White } \\
(91 \%) \text {, male }(65 \%), \\
\text { and had less than a } \\
\text { high school educa- } \\
\text { tion (mean years of } \\
\text { education was 11.9). } \\
\text { Over } 90 \% \text { of partici- } \\
\text { pants were polysub- } \\
\text { stance users, and } \\
\text { average length of } \\
\text { stay at the treat- } \\
\text { ment facility was } 41 \\
\text { (26.2) days }\end{array}$ & $\begin{array}{l}\text { during treat- } \\
\text { ment, 1- and } \\
6 \text { - month post- } \\
\text { treatment } \\
\text { assessments. }\end{array}$ & $\begin{array}{l}\text { and substance use } \\
\text { (SFS). }\end{array}$ & $\begin{array}{l}\text { immediate } \\
\text { increases in } \\
\text { substance use } \\
\text { that peaked and } \\
\text { subsequently } \\
\text { plateaued } \\
\text { around } 13 \\
\text { weeks after } \\
\text { treatment. The } \\
\text { MBRP group's } \\
\text { substance-use } \\
\text { trajectories dif- } \\
\text { fered markedly. } \\
\text { Participants re- } \\
\text { ported statisti- } \\
\text { cally significant } \\
\text { declines in their } \\
\text { level of craving } \\
\text { during the treat- } \\
\text { ment phase. } \\
\text { These declines } \\
\text { were statistically } \\
\text { identical across } \\
\text { treatment con- } \\
\text { ditions. Those } \\
\text { assigned to the } \\
\text { MBRP condition } \\
\text { largely main- } \\
\text { tained their low } \\
\text { levels of craving } \\
\text { throughout the } \\
\text { remainder of } \\
\text { the } 28-\text { week } \\
\text { study period, } \\
\text { those assigned } \\
\text { to the TAU con- } \\
\text { dition showed } \\
\text { rather immedi- } \\
\text { ate and substan- } \\
\text { tial increases in } \\
\text { their craving } \\
\text { levels, before } \\
\text { plateauing ap- } \\
\text { proximately } 14 \\
\text { weeks post- } \\
\text { treatment. Par- } \\
\text { ticipants in } \\
\text { MBPR showed } \\
\text { statistically sig- } \\
\text { nificant im- } \\
\text { provements in } \\
\text { their stress } \\
\text { levels during } \\
\text { the } 8 \text {-week } \\
\text { intervention. }\end{array}$ & $\begin{array}{l}\text { evidence and } \\
\text { support for the } \\
\text { use of MBRP } \\
\text { among high } \\
\text { risk, } \\
\text { marginalized } \\
\text { young adults in } \\
\text { residential } \\
\text { substance use } \\
\text { disorder } \\
\text { treatment. }\end{array}$ \\
\hline $\begin{array}{l}\text { Davis et } \\
\text { at., } 2019 \\
{[34]}\end{array}$ & $\begin{array}{l}\text { Rolling MBRP + } \\
\text { treatment } \\
\text { normally } \\
\text { provided by } \\
\text { the residential } \\
\text { facility: } 8,90- \\
\text { min classes } \\
\text { that were held } \\
\text { twice weekly. } \\
\text { Members were } \\
\text { enrolled as } \\
\text { they entered }\end{array}$ & $\begin{array}{l}\text { TAU + treatment } \\
\text { normally } \\
\text { provided by the } \\
\text { residential facility: } \\
8,90 \text {-min sessions } \\
\text { held twice weekly } \\
\text { that were social } \\
\text { support groups } \\
\text { (Alcoholics and } \\
\text { Narcotics } \\
\text { Anonymous). }\end{array}$ & $\begin{array}{l}79 \text { young adults } \\
\text { (MBRP = 44; TAU = } \\
35) \text { enrolled in a } \\
\text { residential SUD } \\
\text { treatment facility. } \\
25.3(2.7) \text { years old, } \\
\text { the majority of } \\
\text { clients were non- } \\
\text { Hispanic White } \\
(91 \%) \text {, male (65\%), } \\
\text { and had less than a } \\
\text { high school }\end{array}$ & $\begin{array}{l}\text { Baseline, bi- } \\
\text { weekly (every } \\
2 \text { weeks) as- } \\
\text { sessments dur- } \\
\text { ing treatment, } \\
\text { 1- and 6- } \\
\text { month post- } \\
\text { treatment } \\
\text { assessments. }\end{array}$ & $\begin{array}{l}\text { Primary outcomes } \\
\text { were impulsivity } \\
\text { (UPPS-P impulsive } \\
\text { behavior scale) and } \\
\text { substance use (SFS). }\end{array}$ & $\begin{array}{l}\text { Participants } \\
\text { receiving MBRP } \\
\text { evidenced } \\
\text { significant } \\
\text { reductions in all } \\
\text { facets of } \\
\text { impulsivity } \\
\text { except for } \\
\text { sensation } \\
\text { seeking during } \\
\text { treatment, and } \\
\text { these reductions }\end{array}$ & $\begin{array}{l}\text { The MBRP } \\
\text { intervention in } \\
\text { the current } \\
\text { study made } \\
\text { use of a rolling } \\
\text { group } \\
\text { admission, } \\
\text { allowing new } \\
\text { patients at the } \\
\text { residential } \\
\text { facility to enter } \\
\text { the group and }\end{array}$ \\
\hline
\end{tabular}


Table 2 Characteristics of Studies Included in Systematic Review (Continued)

\begin{tabular}{|c|c|c|c|c|c|c|c|}
\hline Reference & $\begin{array}{l}\text { Treatment } \\
\text { condition }\end{array}$ & $\begin{array}{l}\text { Control } \\
\text { condition(s) }\end{array}$ & Overview of sample & $\begin{array}{l}\text { Data collection } \\
\text { time points }\end{array}$ & Outcome measures & Results & Limitations \\
\hline & $\begin{array}{l}\text { the residential } \\
\text { facility (as } \\
\text { opposed to the } \\
\text { standard 8- } \\
\text { week cohort- } \\
\text { based proto- } \\
\text { col). The treat- } \\
\text { ment normally } \\
\text { provided was a } \\
\text { combination of } \\
\text { cognitive- } \\
\text { behavioral } \\
\text { treatment and } \\
\text { the } 12-\text { step ap- } \\
\text { proach to } \\
\text { recovery. }\end{array}$ & & $\begin{array}{l}\text { education (mean } \\
\text { years of education } \\
\text { was 11.9). Over } 90 \% \\
\text { of participants were } \\
\text { polysubstance users, } \\
\text { and average length } \\
\text { of stay at the treat- } \\
\text { ment facility was } 41 \\
\text { (26.2) days. }\end{array}$ & & & $\begin{array}{l}\text { were } \\
\text { significantly } \\
\text { greater than } \\
\text { TAU for all } \\
\text { facets except } \\
\text { positive } \\
\text { urgency. These } \\
\text { treatment gains } \\
\text { were } \\
\text { maintained } \\
\text { through the 6- } \\
\text { month follow- } \\
\text { up. }\end{array}$ & $\begin{array}{l}\text { departing } \\
\text { patients to } \\
\text { leave it. This } \\
\text { may be a } \\
\text { limitation } \\
\text { because } \\
\text { patients could } \\
\text { receive } \\
\text { instruction out } \\
\text { of order, } \\
\text { weakening the } \\
\text { integrity of the } \\
\text { treatment. }\end{array}$ \\
\hline $\begin{array}{l}\text { Glasner- } \\
\text { Edwards } \\
\text { et al., } 2017 \\
\text { [35] }\end{array}$ & $\begin{array}{l}\text { MBRP + } \\
\text { contingency } \\
\text { management } \\
\text { (CM): 8, 75- } \\
\text { min sessions. } \\
\text { Modified } \\
\text { sessions from } \\
120 \text { min to } \\
\text { increase } \\
\text { engagement } \\
\text { by shortening } \\
\text { meditation } \\
\text { practices. All } \\
\text { participants in } \\
\text { the trial also } \\
\text { received CM, } \\
\text { which } \\
\text { consisted of } \\
\text { twice-weekly } \\
\text { visits of the } \\
\text { fishbowl } \\
\text { method, with } \\
\text { rewards based } \\
\text { on urine drug } \\
\text { screes. }\end{array}$ & $\begin{array}{l}\text { Health education } \\
(\mathrm{HE})+ \\
\text { contingency } \\
\text { management } \\
\text { (CM): 8, 75-min } \\
\text { sessions. Ad- } \\
\text { dressed } 6 \text { differ- } \\
\text { ent types of } \\
\text { health (e.g., intel- } \\
\text { lectual, social, } \\
\text { emotional, phys- } \\
\text { ical, environmen- } \\
\text { tal, spiritual). All } \\
\text { participants in } \\
\text { this condition } \\
\text { also received } \\
\text { twice-weekly CM } \\
\text { visits. }\end{array}$ & $\begin{array}{l}63 \text { adults (MBRP + } \\
\mathrm{CM}=31 ; \mathrm{HE}+ \\
\mathrm{CM}=32) \text { with DSM- } \\
\text { IV stimulant de- } \\
\text { pendence recruited } \\
\text { from the commu- } \\
\text { nity. } 71.4 \% \text { male, } \\
45.3(8.9) \text { years old, } \\
30.2 \% \text { non-Hispanic } \\
\text { white, } 43 \% \text { with a } \\
\text { co-occurring Axis } 1 \\
\text { disorder. }\end{array}$ & $\begin{array}{l}\text { Baseline and 1- } \\
\text { month post- } \\
\text { treatment fol- } \\
\text { low- up. }\end{array}$ & $\begin{array}{l}\text { Substance use } \\
\text { outcomes were } \\
\text { stimulant-positive } \\
\text { urine drug screens } \\
\text { and the ASI addic- } \\
\text { tion severity score. } \\
\text { Psychiatric severity } \\
\text { outcomes were the } \\
\text { BDI, BAl, and ASI } \\
\text { psychiatric severity } \\
\text { score. } \\
\text { Hypothesized } \\
\text { mediators were } \\
\text { emotion regulation } \\
\text { (DERS), thought } \\
\text { suppression (WBSI), } \\
\text { and mindfulness } \\
\text { skills (FFMQ). }\end{array}$ & $\begin{array}{l}\text { There was no } \\
\text { effect of } \\
\text { treatment } \\
\text { condition on } \\
\text { odds of } \\
\text { producing a } \\
\text { stimulant- } \\
\text { positive urine } \\
\text { drug screen } \\
\text { over the 8-week } \\
\text { intervention } \\
\text { phase (73\% in } \\
\text { MBRP + CM vs. } \\
\text { 70\% in HE + } \\
\text { CM) or change } \\
\text { in ASI addiction } \\
\text { severity index } \\
\text { scores from } \\
\text { baseline to the } \\
\text { 1-month follow- } \\
\text { up. } \\
\text { However, those } \\
\text { with co- } \\
\text { occurring psy- } \\
\text { chiatric disor- } \\
\text { ders had lower } \\
\text { odds of produ- } \\
\text { cing a } \\
\text { stimulant- } \\
\text { positive urine } \\
\text { drug screen if } \\
\text { they received } \\
\text { MBRP + CM vs. } \\
\text { HE + CM. MBRP } \\
+ \text { CM was asso- } \\
\text { ciated with } \\
\text { greater de- } \\
\text { creases in BDI } \\
\text { scores and in- } \\
\text { creases in ASI } \\
\text { psychiatric se- } \\
\text { verity scores, } \\
\text { but not BAl } \\
\text { scores, as com- } \\
\text { pared to HE + } \\
\text { CM. There was } \\
\text { no significant } \\
\text { group x time } \\
\text { interaction for } \\
\text { any of the puta- } \\
\text { tive }\end{array}$ & $\begin{array}{l}\text { Short follow- } \\
\text { up. High attri- } \\
\text { tion rates ( } 23 \\
\text { of the } 63 \text { par- } \\
\text { ticipants termi- } \\
\text { nated study } \\
\text { involvement). } \\
\text { Shortened } \\
\text { MBRP protocol. }\end{array}$ \\
\hline
\end{tabular}


Table 2 Characteristics of Studies Included in Systematic Review (Continued)

\begin{tabular}{|c|c|c|c|c|c|c|c|}
\hline Reference & $\begin{array}{l}\text { Treatment } \\
\text { condition }\end{array}$ & $\begin{array}{l}\text { Control } \\
\text { condition(s) }\end{array}$ & Overview of sample & $\begin{array}{l}\text { Data collection } \\
\text { time points }\end{array}$ & Outcome measures & Results & Limitations \\
\hline & & & & & & $\begin{array}{l}\text { mechanisms of } \\
\text { change (DERS, } \\
\text { WBSI, FFMQ) } \\
\text { over the study } \\
\text { period. }\end{array}$ & \\
\hline $\begin{array}{l}\text { Hsiao } \\
\text { et al., } 2019 \\
\text { [36] }\end{array}$ & $\begin{array}{l}\text { MBRP: } 8,120- \\
\text { min sessions }\end{array}$ & $\begin{array}{l}\text { TAU: 90-min ses- } \\
\text { sions } 1-2 x / \text { week } \\
\text { during the inter- } \\
\text { vention phase. } \\
\text { Twelve-step, } \\
\text { process-oriented } \\
\text { format. }\end{array}$ & $\begin{array}{l}\text { Adults (study } 1 n= \\
168, \text { study } 2 n= \\
\text { 198) recruited from } \\
\text { an outpatient SUD } \\
\text { treatment program } \\
\text { who had completed } \\
\text { inpatient or } \\
\text { intensive outpatient } \\
\text { treatment in the } \\
\text { previous two weeks. } \\
\text { Study 1: 63.7\% male, } \\
40.5 \text { (10.3) years old, } \\
53.6 \% \text { non-Hispanic } \\
\text { white. Study 2: } \\
75.1 \% \text { male, } 38.2 \\
\text { (10.9) years old, } \\
52.8 \% \text { non-Hispanic } \\
\text { white. }\end{array}$ & $\begin{array}{l}\text { Study 1: } \\
\text { Baseline, post- } \\
\text { treatment, 2- } \\
\text { and 4-month } \\
\text { follow-up } \\
\text { Study 2: Base- } \\
\text { line, post- } \\
\text { treatment, 2-, } \\
\text { 4-, 6-, and 12- } \\
\text { month follow- } \\
\text { up. }\end{array}$ & $\begin{array}{l}\text { Substance use } \\
\text { outcome was } \\
\text { craving (PACS). } \\
\text { Examined a latent } \\
\text { mindfulness factor as } \\
\text { a mediator (AAQ } \\
\text { and acting with } \\
\text { awareness and } \\
\text { nonjudgment } \\
\text { subscales of the } \\
\text { FFMQ). }\end{array}$ & $\begin{array}{l}\text { Study 1: The } \\
\text { effects of MBRP, } \\
\text { as compared to } \\
\text { TAU, on AAQ, } \\
\text { FFMQ, and } \\
\text { PACS scores at } \\
\text { post-treatment } \\
\text { were small-to- } \\
\text { medium } \\
\text { (Cohen's d } \\
\text { range from } 0.08 \\
\text { to 0.48). The la- } \\
\text { tent mindful- } \\
\text { ness factor } \\
\text { significantly me- } \\
\text { diated the ef- } \\
\text { fects of MBRP, } \\
\text { as compared to } \\
\text { TAU, on lower } \\
\text { craving scores } \\
\text { post-treatment. } \\
\text { Study } 2: \text { The } \\
\text { effects of } \\
\text { treatment } \\
\text { condition on } \\
\text { AAQ, FFMQ, and } \\
\text { PACS scores at } \\
\text { post-treatment } \\
\text { were very small } \\
\text { (Cohen's d } \\
\text { range from } 0.03 \\
\text { to } 0.21 \text { and in- } \\
\text { dicated that } \\
\text { those who re- } \\
\text { ceived TAU re- } \\
\text { ported higher } \\
\text { mindfulness } \\
\text { scores than } \\
\text { those who re- } \\
\text { ceived MBRP. } \\
\text { Higher post- } \\
\text { treatment mind- } \\
\text { fulness was as- } \\
\text { sociated with } \\
\text { lower post- } \\
\text { treatment crav- } \\
\text { ing, but those in } \\
\text { the MBRP con- } \\
\text { dition did not } \\
\text { have greater } \\
\text { post-treatment } \\
\text { mindfulness } \\
\text { scores, and } \\
\text { therefore there } \\
\text { was not signifi- } \\
\text { cant mediation. }\end{array}$ & $\begin{array}{l}\text { Low reliability } \\
\text { of the AAQ. } \\
\text { Study } 2 \text { was } \\
\text { conducted } \\
\text { several years } \\
\text { after study } 1 \\
\text { from the same } \\
\text { treatment } \\
\text { program, and } \\
\text { therefore the } \\
\text { treatment } \\
\text { program might } \\
\text { have } \\
\text { integrated } \\
\text { components of } \\
\text { MBls in TAU, } \\
\text { mitigating the } \\
\text { effect of MBRP. } \\
\text { The latent } \\
\text { mindfulness } \\
\text { factor was not } \\
\text { invariant across } \\
\text { the two } \\
\text { samples, } \\
\text { indicating that } \\
\text { the } \\
\text { measurement } \\
\text { of mindfulness } \\
\text { was not } \\
\text { equivalent } \\
\text { across studies. }\end{array}$ \\
\hline $\begin{array}{l}\text { Greenfield } \\
\text { et al., } 2018 \\
\text { [37] }\end{array}$ & $\begin{array}{l}\text { MBRP: } 8,120- \\
\text { min sessions }\end{array}$ & $\begin{array}{l}\text { Relapse } \\
\text { prevention (RP): 8, } \\
\text { 120-min sessions. } \\
\text { Topics included } \\
\text { self-efficacy, }\end{array}$ & $\begin{array}{l}191 \text { adults with SUD } \\
\text { who were recruited } \\
\text { following inpatient } \\
\text { or intensive } \\
\text { outpatient }\end{array}$ & $\begin{array}{l}\text { Baseline and } \\
12 \text { - month } \\
\text { post-treatment } \\
\text { follow- up. }\end{array}$ & $\begin{array}{l}\text { Number of drug use } \\
\text { days and number of } \\
\text { heavy drinking days } \\
\text { in the } 90 \text {-day period } \\
\text { before the 12- }\end{array}$ & $\begin{array}{l}\text { Among racial/ } \\
\text { ethnic minority } \\
\text { participants, } \\
\text { there was not a } \\
\text { significant }\end{array}$ & $\begin{array}{l}\text { Not able to } \\
\text { examine } \\
\text { particular } \\
\text { racial/ethnic } \\
\text { minority }\end{array}$ \\
\hline
\end{tabular}


Table 2 Characteristics of Studies Included in Systematic Review (Continued)

\begin{tabular}{|c|c|c|c|c|c|c|c|}
\hline Reference & $\begin{array}{l}\text { Treatment } \\
\text { condition }\end{array}$ & $\begin{array}{l}\text { Control } \\
\text { condition(s) }\end{array}$ & Overview of sample & $\begin{array}{l}\text { Data collection } \\
\text { time points }\end{array}$ & Outcome measures & Results & Limitations \\
\hline & & $\begin{array}{l}\text { coping skills, goal } \\
\text { setting, problem } \\
\text { solving, and social } \\
\text { support. }\end{array}$ & $\begin{array}{l}\text { treatment. } 71.0 \% \\
\text { male, } 39.04(10.93) \\
\text { years old, } 52.9 \% \\
\text { non-Hispanic white. } \\
43.5 \% \text { of partici- } \\
\text { pants were in } \\
\text { groups comprised } \\
\text { of }>50 \% \text { non- } \\
\text { Hispanic white par- } \\
\text { ticipants and } 56.5 \% \\
\text { were in the groups } \\
\text { comprised of }>50 \% \\
\text { racial/ethnic minor- } \\
\text { ity participants. }\end{array}$ & & $\begin{array}{l}\text { month post- } \\
\text { treatment follow-up. }\end{array}$ & $\begin{array}{l}\text { difference in } \\
\text { heavy drinking } \\
\text { days between } \\
\text { MBRP and RP, } \\
\text { but those who } \\
\text { received MBRP } \\
\text { reported fewer } \\
\text { drug use days } \\
\text { than those who } \\
\text { received RP. } \\
\text { Among non- } \\
\text { Hispanic white } \\
\text { participants, } \\
\text { those who re- } \\
\text { ceived MBRP re- } \\
\text { ported fewer } \\
\text { heavy drinking } \\
\text { days, as com- } \\
\text { pared to those } \\
\text { who received } \\
\text { RP, but there } \\
\text { was no differ- } \\
\text { ence in drug } \\
\text { use days by } \\
\text { treatment con- } \\
\text { dition. Among } \\
\text { individuals in } \\
\text { groups com- } \\
\text { prised of }>50 \% \\
\text { racial/ethnic mi- } \\
\text { nority partici- } \\
\text { pants, there was } \\
\text { not a significant } \\
\text { difference in } \\
\text { heavy drinking } \\
\text { days between } \\
\text { MBRP and RP. } \\
\text { Among those in } \\
\text { groups with > } \\
50 \% \text { non- } \\
\text { Hispanic white } \\
\text { participants, } \\
\text { those who re- } \\
\text { ceived MBRP } \\
\text { had fewer heavy } \\
\text { drinking days } \\
\text { than those who } \\
\text { received RP. } \\
\text { There was not a } \\
\text { significant inter- } \\
\text { action between } \\
\text { group racial/eth- } \\
\text { nic composition } \\
\text { and treatment } \\
\text { condition in } \\
\text { predicting drug } \\
\text { use days. In sub- } \\
\text { group analyses } \\
\text { of only racial/ } \\
\text { ethnic minority } \\
\text { individuals, } \\
\text { there was a sig- } \\
\text { nificant inter- } \\
\text { action between } \\
\text { group race/eth- } \\
\text { nicity compos- } \\
\text { ition and }\end{array}$ & $\begin{array}{l}\text { groups or } \\
\text { differences by } \\
\text { acculturation/ } \\
\text { racial identity. }\end{array}$ \\
\hline
\end{tabular}


Table 2 Characteristics of Studies Included in Systematic Review (Continued)

\begin{tabular}{|c|c|c|c|c|c|c|c|}
\hline Reference & $\begin{array}{l}\text { Treatment } \\
\text { condition }\end{array}$ & $\begin{array}{l}\text { Control } \\
\text { condition(s) }\end{array}$ & Overview of sample & $\begin{array}{l}\text { Data collection } \\
\text { time points }\end{array}$ & Outcome measures & Results & Limitations \\
\hline & & & & & & $\begin{array}{l}\text { treatment con- } \\
\text { dition in pre- } \\
\text { dicting heavy } \\
\text { drinking days, } \\
\text { but not drug } \\
\text { use days. } \\
\text { Among racial/ } \\
\text { ethnic minority } \\
\text { individuals in } \\
\text { groups com- } \\
\text { prised of }>50 \% \\
\text { racial/ethnic mi- } \\
\text { norities, MBRP } \\
\text { produced fewer } \\
\text { heavy drinking } \\
\text { days than RP. } \\
\text { There was no } \\
\text { difference in } \\
\text { heavy drinking } \\
\text { days by treat- } \\
\text { ment condition } \\
\text { for racial/ethnic } \\
\text { minority individ- } \\
\text { uals who were } \\
\text { in groups with } \\
>50 \% \text { whites. In } \\
\text { subgroup ana- } \\
\text { lyses of only } \\
\text { non-Hispanic } \\
\text { white }\end{array}$ & \\
\hline $\begin{array}{l}\text { Roos et al., } \\
2017 \text { [38] }\end{array}$ & $\begin{array}{l}\text { MBRP: } 8,120- \\
\text { min sessions. }\end{array}$ & $\begin{array}{l}\text { TAU: 12-step fa- } \\
\text { cilitation and } \\
\text { process-oriented } \\
\text { groups. Met } 1-2 \times / \\
\text { wk. for } 1.5 \mathrm{~h} \text {. } \\
\text { RP: (study } 1 \text { only) } \\
8,120-\text { min group } \\
\text { sessions. Treat- } \\
\text { ment match con- } \\
\text { trol for MBRP }\end{array}$ & $\begin{array}{l}454 \text { adults (study } 1 \\
n=286, \text { study } 2 n= \\
\text { 168) recruited from } \\
\text { an outpatient SUD } \\
\text { treatment program } \\
\text { who had completed } \\
\text { inpatient or } \\
\text { intensive outpatient } \\
\text { treatment in the } \\
\text { previous two weeks. } \\
\text { Study 1: } 71.8 \% \text { male, } \\
38.4 \text { (10.9) years old, } \\
51.6 \% \text { non-Hispanic } \\
\text { white. Study 2: } \\
63.7 \% \text { male, } 40.5 \\
\text { (10.3) years old, } \\
53.6 \% \text { non-Hispanic } \\
\text { white. }\end{array}$ & $\begin{array}{l}\text { Study 1: } \\
\text { Baseline, post- } \\
\text { treatment, 3-, } \\
\text { 6-, 12-month } \\
\text { follow-up } \\
\text { Study 2: Base- } \\
\text { line, post- } \\
\text { treatment, 2- } \\
\text { and 4-month } \\
\text { follow-up. }\end{array}$ & $\begin{array}{l}\text { Alcohol and drug } \\
\text { use days were } \\
\text { assessed using the } \\
\text { TLFB. Substance use } \\
\text { disorder symptom } \\
\text { severity was } \\
\text { measured using the } \\
\text { Severity and } \\
\text { Dependence Scale } \\
\text { (SDS) and the Short } \\
\text { Inventory of } \\
\text { Problems (SIP). } \\
\text { Depression and } \\
\text { anxiety symptoms } \\
\text { were measured } \\
\text { using the BDI-II and } \\
\text { BAI, respectively }\end{array}$ & $\begin{array}{l}\text { Study 1: } \\
\text { Individuals with } \\
\text { high SUD } \\
\text { severity and } \\
\text { high } \\
\text { depression/ } \\
\text { anxiety and in } \\
\text { the MBRP } \\
\text { condition } \\
\text { experienced } \\
\text { significantly } \\
\text { fewer heavy } \\
\text { drinking days } \\
\text { (HDD) and drug } \\
\text { use days (DUD) } \\
\text { at } 12-\text {-month } \\
\text { follow-up rela- } \\
\text { tive to RP and } \\
\text { TAU. No effects } \\
\text { were seen in } \\
\text { low SUD sever- } \\
\text { ity and low de- } \\
\text { pression/anxiety } \\
\text { class. High SUD } \\
\text { severity and low } \\
\text { depression/anx- } \\
\text { iety and MBRP } \\
\text { condition pre- } \\
\text { dicted signifi- } \\
\text { cantly fewer 12- } \\
\text { month HDD } \\
\text { relative to RP or } \\
\text { TAU. Study 2: } \\
\text { MBRP condition } \\
\text { for high severity } \\
\text { and high de- } \\
\text { pression/anxiety }\end{array}$ & $\begin{array}{l}\text { A retrospective } \\
\text { self- report } \\
\text { measure of } \\
\text { days abstinent } \\
\text { was used, } \\
\text { which may not } \\
\text { accurately } \\
\text { reflect actual } \\
\text { alcohol and } \\
\text { drug use days. }\end{array}$ \\
\hline
\end{tabular}


Table 2 Characteristics of Studies Included in Systematic Review (Continued)

\begin{tabular}{|c|c|c|c|c|c|c|c|}
\hline Reference & $\begin{array}{l}\text { Treatment } \\
\text { condition }\end{array}$ & $\begin{array}{l}\text { Control } \\
\text { condition(s) }\end{array}$ & Overview of sample & $\begin{array}{l}\text { Data collection } \\
\text { time points }\end{array}$ & Outcome measures & Results & Limitations \\
\hline & & & & & & $\begin{array}{l}\text { and high sever- } \\
\text { ity SUD and low } \\
\text { depression/anx- } \\
\text { iety had signifi- } \\
\text { cantly fewer } \\
\text { alcohol and } \\
\text { other drug use } \\
\text { days relative to } \\
\text { TAU. }\end{array}$ & \\
\hline $\begin{array}{l}\text { Roos et al., } \\
2019 \text { [39] }\end{array}$ & $\begin{array}{l}\text { MBRP: } 8,120- \\
\text { min sessions }\end{array}$ & $\begin{array}{l}\text { Relapse } \\
\text { prevention (RP): 8, } \\
\text { 120-min sessions. } \\
\text { Topics included } \\
\text { self-efficacy, cop- } \\
\text { ing skills, goal set- } \\
\text { ting, problem } \\
\text { solving, and social } \\
\text { support. }\end{array}$ & $\begin{array}{l}117 \text { adults recruited } \\
\text { from an outpatient } \\
\text { SUD treatment } \\
\text { program who had } \\
\text { completed inpatient } \\
\text { or intensive } \\
\text { outpatient } \\
\text { treatment in the } \\
\text { previous two weeks. } \\
70.9 \% \text { male, } 53.8 \% \\
\text { non-Hispanic white, } \\
\text { 39.0(10.9) years old. }\end{array}$ & $\begin{array}{l}\text { 12-month } \\
\text { follow- up }\end{array}$ & $\begin{array}{l}\text { Substance use was } \\
\text { measured using the } \\
\text { TLFB. Severity of } \\
\text { substance use } \\
\text { disorder was } \\
\text { measured using the } \\
\text { Severity and } \\
\text { Dependence Scale. }\end{array}$ & $\begin{array}{l}\text { Condition } \\
\text { predicted fewer } \\
\text { drug use days } \\
\text { (DUD) such that } \\
\text { MBRP } \\
\text { participants } \\
\text { reported 84\% } \\
\text { fewer DUD than } \\
\text { RP participants. } \\
\text { Individual } \\
\text { gender and } \\
\text { gender group } \\
\text { composition not } \\
\text { a significant } \\
\text { predictor of } \\
\text { heavy drinking } \\
\text { days (HDD). } \\
\text { There was a } \\
\text { significant } \\
\text { interaction } \\
\text { between } \\
\text { treatment } \\
\text { condition and } \\
\text { gender } \\
\text { composition on } \\
\text { DUD at } 12- \\
\text { month follow- } \\
\text { up such that } \\
\text { MBRP partici- } \\
\text { pants had fewer } \\
12-\text {-month DUD } \\
\text { than those re- } \\
\text { ceiving RP and } \\
\text { was most pro- } \\
\text { nounced } \\
\text { among groups } \\
\text { comprised of } \\
\text { one-third or } \\
\text { more women. }\end{array}$ & $\begin{array}{l}\text { Only a single } \\
\text { follow-up 12- } \\
\text { months after } \\
\text { the completion } \\
\text { of the } \\
\text { intervention. }\end{array}$ \\
\hline $\begin{array}{l}\text { Shorey } \\
\text { et al., } 2016 \\
\text { [40] }\end{array}$ & $\begin{array}{l}\text { MBRP }+ \\
\text { Acceptance } \\
\text { and } \\
\text { Commitment } \\
\text { Therapy (ACT): } \\
8 \text { bi-weekly, } 90 \\
\text { min sessions. } \\
20-30 \text { min of } \\
\text { guided medita- } \\
\text { tion followed } \\
\text { by experiential } \\
\text { activities and } \\
\text { discussion. } \\
\text { Daily }\end{array}$ & $\begin{array}{l}\text { TAU: Program } \\
\text { guided by a 12- } \\
\text { step model. } \\
\text { Varied daily } \\
\text { therapeutic } \\
\text { activities } \\
\text { including: process } \\
\text { groups, AA/NA } \\
\text { meetings, coping } \\
\text { skills groups, } \\
\text { family therapy, } \\
\text { exercise groups, } \\
\text { acupuncture, } \\
\text { individual session }\end{array}$ & $\begin{array}{l}117 \text { adults }(\mathrm{MBI}=64 ; \\
\text { TAU }=53) \text { in } \\
\text { residential } \\
\text { substance use } \\
\text { disorder treatment } \\
\text { ( } 28-30 \text { day } \\
\text { program). } 74.3 \% \\
\text { male, } 92.2 \% \text { non- } \\
\text { Hispanic White, } 41.3 \\
\text { (10.7) years old. }\end{array}$ & $\begin{array}{l}\text { Baseline and } \\
\text { post-treatment } \\
\text { (at discharge) }\end{array}$ & $\begin{array}{l}\text { Primary outcomes } \\
\text { were: craving (PACS), } \\
\text { mindfulness (FFMQ), } \\
\text { and psychological } \\
\text { flexibility (AAQ-SA). }\end{array}$ & $\begin{array}{l}\text { No significant } \\
\text { difference } \\
\text { between } \\
\text { conditions at } \\
\text { discharge. Effect } \\
\text { sizes between } \\
\text { groups were } \\
\text { small. MBRP + } \\
\text { ACT participants } \\
\text { reported lower } \\
\text { craving and } \\
\text { higher } \\
\text { psychological } \\
\text { flexibility. }\end{array}$ & $\begin{array}{l}\text { Participants } \\
\text { only attended } \\
\text { an average of } \\
5.4 \text { of the } 8 \\
\text { classes in the } \\
\text { MBRP + ACT } \\
\text { group. } \\
\text { Attendance } \\
\text { was not } \\
\text { tracked in the } \\
\text { TAU condition. }\end{array}$ \\
\hline
\end{tabular}


Table 2 Characteristics of Studies Included in Systematic Review (Continued)

\begin{tabular}{|c|c|c|c|c|c|c|c|}
\hline Reference & $\begin{array}{l}\text { Treatment } \\
\text { condition }\end{array}$ & $\begin{array}{l}\text { Control } \\
\text { condition(s) }\end{array}$ & Overview of sample & $\begin{array}{l}\text { Data collection } \\
\text { time points }\end{array}$ & Outcome measures & Results & Limitations \\
\hline & $\begin{array}{l}\text { normally would } \\
\text { have attended } \\
\text { process groups. }\end{array}$ & & & & & & \\
\hline $\begin{array}{l}\text { Weiss de } \\
\text { Souza } \\
\text { et al., } 2020 \\
{[41]}\end{array}$ & $\begin{array}{l}\text { MBRP + The } \\
\text { Brazilian } \\
\text { Ministry of } \\
\text { Health's } \\
\text { tobacco } \\
\text { cessation } \\
\text { standard } \\
\text { treatment (ST) } \\
\text { protocol: } 8 \text {, } \\
\text { 120- min } \\
\text { sessions. The } \\
\text { ST protocol } \\
\text { was a } \\
\text { cognitive- } \\
\text { behavioral } \\
\text { treatment } \\
\text { delivered in } \\
\text { four structured } \\
\text { weekly sessions } \\
\text { and six } \\
\text { maintenance } \\
\text { follow-up ses- } \\
\text { sions. Sessions } \\
\text { were designed } \\
\text { to provide in- } \\
\text { formation on } \\
\text { risks of smok- } \\
\text { ing and bene- } \\
\text { fits of quitting, } \\
\text { stimulate self- } \\
\text { control and } \\
\text { self- } \\
\text { management } \\
\text { to disrupt the } \\
\text { cycle of de-- } \\
\text { pendence, and } \\
\text { support } \\
\text { smokers to be- } \\
\text { come agents of } \\
\text { change con- } \\
\text { cerning their } \\
\text { behavior. All } \\
\text { participants re- } \\
\text { ceived } 4,90- \\
\text { min structured } \\
\text { weekly sessions } \\
\text { during the } \\
\text { smoking cessa- } \\
\text { tion phase of } \\
\text { ST, and 6, } 90- \\
\text { min ST main- } \\
\text { tenance ses- } \\
\text { sions (at Weeks } \\
6,8,10,12,24, \\
\text { and } 48 \text { ) }\end{array}$ & $\begin{array}{l}\text { ST: } 4,90 \text {-min } \\
\text { structured weekly } \\
\text { sessions during } \\
\text { the smoking ces- } \\
\text { sation phase, and } \\
6,90 \text {-min main- } \\
\text { tenance sessions } \\
\text { (at Weeks } 6,8,10 \text {, } \\
12,24 \text {, and } 48 \text { ) }\end{array}$ & $\begin{array}{l}86 \text { individuals (ST } \\
n=42 ; \text { MBRP } n=44) \\
\text { recruited by phone } \\
\text { from a waiting list } \\
\text { of an outpatient } \\
\text { public health } \\
\text { tobacco treatment } \\
\text { service in a single } \\
\text { city in Brazil. } 50.34 \\
(10.25) \text { years old, } \\
80.23 \% \text { female, and } \\
60.46 \% \text { smoked over } \\
10 \text { cigarettes per } \\
\text { day. }\end{array}$ & $\begin{array}{l}\text { Baseline, 4-, } \\
\text { 12-, and 24- } \\
\text { week follow- } \\
\text { ups. }\end{array}$ & $\begin{array}{l}\text { The primary } \\
\text { outcome was } \\
\text { smoking abstinence } \\
\text { (expired carbon } \\
\text { monoxide, } \\
\text { participants with a } \\
\text { carbon monoxide of } \\
<10 \text { were } \\
\text { considered } \\
\text { abstinent). } \\
\text { Secondary outcomes } \\
\text { included levels of } \\
\text { mindfulness (FFMQ- } \\
\text { BR), craving (QSU), } \\
\text { positive and } \\
\text { negative affect } \\
\text { (PANAS), and } \\
\text { depression and } \\
\text { anxiety (HAD Scale). }\end{array}$ & 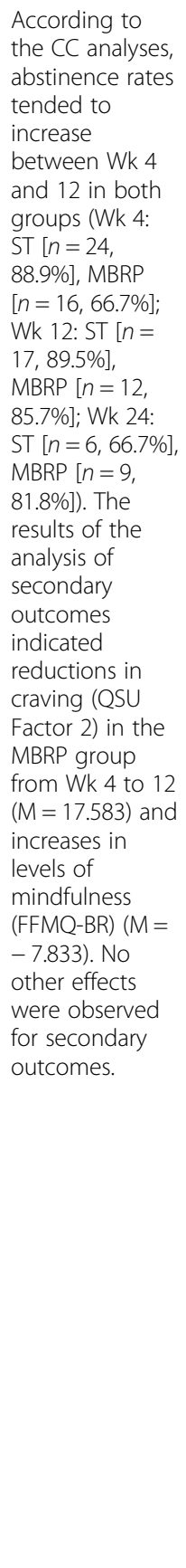 & $\begin{array}{l}\text { Small sample } \\
\text { size with large } \\
\text { attrition rates } \\
\text { through the } \\
\text { 24-week } \\
\text { follow-up in } \\
\text { both groups } \\
\text { (MPRB - 75\%; } \\
\text { ST - 78.57\%). }\end{array}$ \\
\hline $\begin{array}{l}\text { Witkiewitz } \\
\text { et al., } 2019 \\
\text { [42] }\end{array}$ & $\begin{array}{l}\text { MBRP + Active } \\
\text { tDCS: } 8,120- \\
\text { min sessions } \\
\text { with the first } \\
\text { 30-min consist- } \\
\text { ing of tDCS. }\end{array}$ & $\begin{array}{l}\text { MBRP + sham } \\
\text { tDCS: Standard } \\
\text { MBRP }+8,120- \\
\text { min sessions with } \\
\text { the first } 30-\text { min } \\
\text { consisting of } \\
\text { tDCS. }\end{array}$ & $\begin{array}{l}134 \text { adults } \\
\text { interested in } \\
\text { reducing their } \\
\text { drinking. } 59.5 \% \\
\text { male, } 47.6 \% \text { non- } \\
\text { Hispanic white, } \\
38.1 \% \text { Hispanic, } 52.3 \\
\text { (13.0) years old. }\end{array}$ & $\begin{array}{l}\text { Baseline, post- } \\
\text { treatment, and } \\
\text { two-month } \\
\text { follow-up }\end{array}$ & $\begin{array}{l}\text { Primary outcomes } \\
\text { were alcohol } \\
\text { consumption (TLFB), } \\
\text { craving (PACS), } \\
\text { alcohol cue-reactivity } \\
\text { (assessed using a vis- } \\
\text { ual cue task), and in- } \\
\text { hibitory control }\end{array}$ & $\begin{array}{l}\text { No difference in } \\
\text { attendance by } \\
\text { condition. } \\
\text { Significant } \\
\text { reductions in } \\
\text { drinks per } \\
\text { drinking day } \\
\text { over time. }\end{array}$ & $\begin{array}{l}\text { Low follow-up } \\
\text { rate. } \\
\text { Exclusion of } \\
\text { individuals with } \\
\text { history } \\
\text { of severe }\end{array}$ \\
\hline
\end{tabular}


Table 2 Characteristics of Studies Included in Systematic Review (Continued)

\begin{tabular}{|c|c|c|c|c|c|c|c|}
\hline Reference & $\begin{array}{l}\text { Treatment } \\
\text { condition }\end{array}$ & $\begin{array}{l}\text { Control } \\
\text { condition(s) }\end{array}$ & Overview of sample & $\begin{array}{l}\text { Data collection } \\
\text { time points }\end{array}$ & Outcome measures & Results & Limitations \\
\hline & & & & & $\begin{array}{l}\text { (assessed using a } \\
\text { stop signal task). }\end{array}$ & $\begin{array}{l}\text { Significant dose } \\
\text { effect for the } \\
\text { number of } \\
\text { groups } \\
\text { attended. } \\
\text { Significant } \\
\text { effects of time } \\
\text { and dose for } \\
\text { the number of } \\
\text { groups } \\
\text { attended on } \\
\text { percent heavy } \\
\text { drinking days } \\
\text { and cue } \\
\text { reactivity. No } \\
\text { effects of active } \\
\text { versus sham } \\
\text { tDCS on } \\
\text { outcomes. }\end{array}$ & $\begin{array}{l}\text { alcohol } \\
\text { withdrawal } \\
\text { likely } \\
\text { reduced } \\
\text { variability in } \\
\text { sample and } \\
\text { likely } \\
\text { reduced } \\
\text { sample AUD } \\
\text { severity. No } \\
\text { biomarkers } \\
\text { of drinking-- } \\
\text { just self- } \\
\text { report. }\end{array}$ \\
\hline $\begin{array}{l}\text { Yaghubi } \\
\text { et al., } 2017 \\
\text { [43] }\end{array}$ & $\begin{array}{l}\text { MBRP: } 8,120- \\
\text { min sessions. }\end{array}$ & $\begin{array}{l}\text { TAU: Standard for } \\
\text { opioid } \\
\text { dependence (no } \\
\text { substantive } \\
\text { description of } \\
\text { treatment } \\
\text { provided) }\end{array}$ & $\begin{array}{l}70 \text { Iranian adults } \\
\text { receiving } \\
\text { methadone for } \\
\text { opioid dependence. } \\
100 \% \text { male, 20-45 } \\
\text { years old. }\end{array}$ & $\begin{array}{l}\text { Baseline, post- } \\
\text { intervention, } \\
\text { and 2-month } \\
\text { follow-up. }\end{array}$ & $\begin{array}{l}\text { Primary outcomes } \\
\text { were impulsive } \\
\text { behavior (BIS-11) } \\
\text { and opioid (assessed } \\
\text { using a biochemical } \\
\text { morphine test). }\end{array}$ & $\begin{array}{l}\text { MBRP predicted } \\
\text { a significantly } \\
\text { higher } \\
\text { likelihood of } \\
\text { abstinence } \\
\text { relative to TAU } \\
\text { at post- } \\
\text { treatment and } \\
\text { follow-up. MBRP } \\
\text { also predicted a } \\
\text { significant de- } \\
\text { crease in impul- } \\
\text { sivity among all } \\
\text { subscales and } \\
\text { total scores at } \\
\text { post-treatment } \\
\text { and follow-up. }\end{array}$ & $\begin{array}{l}\text { The study may } \\
\text { have limited } \\
\text { generalizability } \\
\text { because the } \\
\text { sample was } \\
\text { entirely male. }\end{array}$ \\
\hline $\begin{array}{l}\text { Zemestani } \\
\text { et al., } 2016 \\
\text { [44] }\end{array}$ & $\begin{array}{l}\text { MBRP: } 8,120- \\
\text { min sessions. }\end{array}$ & $\begin{array}{l}\text { TAU: 12-step, } \\
\text { process oriented } \\
\text { inpatient } \\
\text { treatment. } \\
\text { Topics included } \\
\text { psycho-education, } \\
\text { effects of sub- } \\
\text { stance use on } \\
\text { interpersonal rela- } \\
\text { tionships, rational } \\
\text { thinking skills, re- } \\
\text { lapse prevention } \\
\text { skills and related } \\
\text { themes. }\end{array}$ & $\begin{array}{l}74 \text { Iranian adults } \\
\text { receiving inpatient } \\
\text { treatment for } \\
\text { substance use } \\
\text { disorder. } 79.7 \% \\
\text { male, } 30.1 \text { (9.4) years } \\
\text { old. }\end{array}$ & $\begin{array}{l}\text { Baseline, post- } \\
\text { intervention, } \\
\text { and 2-month } \\
\text { follow-up. }\end{array}$ & $\begin{array}{l}\text { Primary outcomes } \\
\text { were depression and } \\
\text { anxiety symptoms } \\
\text { (BDI-II and the BAI) } \\
\text { and craving (PACS). }\end{array}$ & $\begin{array}{l}\text { Participants in } \\
\text { the MBRP } \\
\text { condition } \\
\text { reported } \\
\text { significantly } \\
\text { lower post- } \\
\text { intervention } \\
\text { rates of depres- } \\
\text { sion, anxiety, } \\
\text { and craving as } \\
\text { compared to } \\
\text { those in TAU. } \\
\text { These results } \\
\text { were consistent } \\
\text { at follow-up. }\end{array}$ & $\begin{array}{l}\text { Many patients } \\
\text { did not meet } \\
\text { criteria for a } \\
\text { major } \\
\text { depressive } \\
\text { disorder }\end{array}$ \\
\hline $\begin{array}{l}\text { Zgierska } \\
\text { et al., } 2019 \\
{[45]}\end{array}$ & $\begin{array}{l}\text { MBRP (tailored } \\
\text { to AUD) + } \\
\text { standard care: } \\
\text { 8, 120-min ses- } \\
\text { sions plus 6+ } \\
\text { days per week } \\
\text { formal (e.g., }\end{array}$ & $\begin{array}{l}\text { TAU: Individual } \\
\text { and/or group } \\
\text { outpatient } \\
\text { therapy for } \\
\text { alcohol use } \\
\text { disorder. } \\
\text { Primary }\end{array}$ & $\begin{array}{l}112 \text { adults receiving } \\
\text { inpatient treatment } \\
\text { for alcohol } \\
\text { dependence. } 56.2 \% \\
\text { male, } 91 \% \text { non- } \\
\text { Hispanic White, } 41 \\
\text { (12.2) years old }\end{array}$ & $\begin{array}{l}\text { Baseline, post- } \\
\text { treatment, and } \\
\text { 18-week } \\
\text { follow-up }\end{array}$ & $\begin{array}{l}\text { Primary outcomes } \\
\text { were: alcohol } \\
\text { consumption (TLFB), } \\
\text { alcohol-related con- } \\
\text { sequences (DrInC), } \\
\text { stress (PSS), and } \\
\text { mindfulness (MAAS). }\end{array}$ & $\begin{array}{l}\text { No significant } \\
\text { differences } \\
\text { between } \\
\text { conditions at } \\
\text { post-treatment } \\
\text { and follow-up. }\end{array}$ & $\begin{array}{l}\text { Small sample } \\
\text { size and there } \\
\text { was no } \\
\text { blinding. Only } \\
\text { one therapist } \\
\text { provided all of } \\
\text { the treatment. }\end{array}$ \\
\hline
\end{tabular}


Table 2 Characteristics of Studies Included in Systematic Review (Continued)

\begin{tabular}{|c|c|c|c|c|c|c|c|}
\hline Reference & $\begin{array}{l}\text { Treatment } \\
\text { condition }\end{array}$ & $\begin{array}{l}\text { Control } \\
\text { condition(s) }\end{array}$ & Overview of sample & $\begin{array}{l}\text { Data collection } \\
\text { time points }\end{array}$ & Outcome measures & Results & Limitations \\
\hline & & $\begin{array}{l}\text { Encouraged to } \\
\text { participate in mu- } \\
\text { tual self-help } \\
\text { meetings }\end{array}$ & & & & & \\
\hline
\end{tabular}

Mindfulness Oriented Recovery Enhancement (MORE)

\begin{tabular}{|c|c|c|c|}
\hline $\begin{array}{l}\text { Garland } \\
\text { et al. 2016 } \\
\text { and } \\
\text { Garland } \\
\text { et al. 2018 } \\
\text { (erratum) } \\
{[15,46]}\end{array}$ & $\begin{array}{l}\text { MORE: } 10,120- \\
\text { min sessions. }\end{array}$ & $\begin{array}{l}\text { TAU: Groups at } \\
\text { the therapeutic } \\
\text { community } \\
\text { (approximately } 2 \\
\text { h/day). Consisted } \\
\text { of } \\
\text { psychoeducation, } \\
\text { coping skills, etc. } \\
\text { CBT: 10, 120-min } \\
\text { group sessions. } \\
\text { Addressed co- } \\
\text { occurring SUD } \\
\text { and PTSD via } \\
\text { skills training (i.e., } \\
\text { not exposure- } \\
\text { based). }\end{array}$ & $\begin{array}{l}180 \text { men with co- } \\
\text { occurring psychiatric } \\
\text { and SUD (MORE = } \\
64, \text { CBT = 64, TAU = } \\
\text { 52) who were previ- } \\
\text { ously homeless, cur- } \\
\text { rently resided in a } \\
\text { therapeutic commu- } \\
\text { nity, and had histor- } \\
\text { ies of trauma. MORE: } \\
37.7 \text { (SD = 10.4) } \\
\text { years old, } 40 \% \text { non- } \\
\text { Hispanic white. CBT: } \\
36.5 \text { (SD = } 11.2 \text { ) } \\
\text { years old, } 44 \% \text { non- } \\
\text { Hispanic White, TAU: } \\
38.7 \text { (SD = 9.8) years } \\
\text { old, 42\% non- } \\
\text { Hispanic white. }\end{array}$ \\
\hline
\end{tabular}

Primary outcomes Craving No postwere craving (PACS), PTSD symptoms $(\mathrm{PCL})$, psychiatric distress (BSI).

Secondary outcomes were mindfulness (FFMQ) and positive and negative affect (PANAS).

Hispanic white.

decreased in all treatment

greater decreases in

MORE, as compared to

CBT, but not as compared to produced

greater ited

decreases in

PTSD symptoms

than CBT and

TAU. Anxiety

and depression

symptoms

decreased treatments, with follow-up as-

TAU. MORE also sessments. Limgeneralizability (i.e., to women, individuals not residing in a

across all

groups, but

there was not a

significant

treatment $x$

time interaction

effect for these

outcomes.

MORE produced

greater

increases in

mindfulness, as

compared to

control

conditions.

MORE produced

greater

increases in

positive affect,

as compared to

TAU, and

decreases in

negative affect,

as compared to

CBT. Changes in

mindfulness

were

significantly

negatively

correlated with

changes in

craving and

PTSD symptoms

and changes in

mindfulness

mediated the

effect of MORE

(vs. control

conditions) on

decreases in

craving and

PTSD community,

etc.)

(n) 
Table 2 Characteristics of Studies Included in Systematic Review (Continued)

\begin{tabular}{|c|c|c|}
\hline Reference & $\begin{array}{l}\text { Treatment } \\
\text { condition }\end{array}$ & $\begin{array}{l}\text { Control } \\
\text { condition(s) }\end{array}$ \\
\hline $\begin{array}{l}\text { Garland } \\
\text { et al., } 2017 \\
\text { [47] }\end{array}$ & $\begin{array}{l}\text { MORE } 8,120- \\
\text { min sessions. }\end{array}$ & $\begin{array}{l}\text { Support group } \\
\text { (SG); } 8,120-\text { min } \\
\text { sessions. The } \\
\text { topics were } \\
\text { pertinent to } \\
\text { chronic pain and } \\
\text { long-term opioid } \\
\text { use and included: } \\
\text { the physical and } \\
\text { emotional aspects } \\
\text { of pain experi- } \\
\text { ence, ways of } \\
\text { coping with } \\
\text { chronic pain, } \\
\text { ways of coping } \\
\text { with negative } \\
\text { emotions, the ef- } \\
\text { fect of life stress } \\
\text { on pain, the } \\
\text { stigma of opioid } \\
\text { use, risks of long- } \\
\text { term opioid use } \\
\text { and opioid de- } \\
\text { pendence, ac- } \\
\text { ceptance versus } \\
\text { denial, and plans } \\
\text { for the future. }\end{array}$ \\
\hline
\end{tabular}

Garland MORE: 8, 120et al., 2017 min sessions. [48]
Overview of sample Data collection

time points
115 prescription

opioid users

(MORE $=57$

randomized; SG =

58) for pain relief on

a daily or near-daily

basis for at least 90

days, and self- re-

ported chronic pain

conditions including

low-back pain, fibro-

myalgia, arthritis,

cervical pain, or

other pain condi-

tions unrelated to

cancer. $67.8 \%$ fe-

male, $48.4(\mathrm{SD}=$

13.6) years, $65 \%$

non- Hispanic white.

\section{Baseline, post- treatment, 3-}

month post-

treatment

follow-up

o

Results

symptoms.

The

outcome was opioid pretreatment

attentional bias (AB) $200 \mathrm{~ms}$ opioid

as measured by the $A B$ status

dot-probe task.

significantly

moderated the

effect of the

treatment

condition on

post-treatment

opioid $A B$, indi-

cating that the

effects of treat-

ment signifi-

cantly differed

by pretreatment opioid $A B$ status.

There were no

significant ef-

fects of the

treatment con-

dition on post-

treatment 2000

ms opioid $A B$

found, nor did

pretreatment

opioid $A B$ serve

as a moderator

of treatment ef-

fects. The 2000

ms opioid $A B$

did not change

from pretreat-

ment to post-

treatment, but

the reductions

in $200 \mathrm{~ms}$ opioid

$A B$ over the

course of treat-

ment predicted

lower levels of

opioid misuse at

3- month

follow-up, sug-

gesting that de-

creasing atten-

tional fixation

on opioid cues

may reduce risk

of future opioid

misuse.

Support group

(SG): 8, 120- min sessions. The topics were pertinent to chronic pain and long-term opioid use and included: the physical and emotional aspects of pain experience, ways of coping with chronic pain,
55 chronic pain patients. This subset was taken from a larger sample (115) during an RCT testing the effectiveness of the MORE intervention for individuals with chronic pain. MORE $n=26$, SG $n=29,21$ men and 34 women, 48.9 (11.6) years old.
Participants completed EMA via a daily diary in which they rated their current pain level and affect rating each time they took their opioid dose with ratings accepted up to four times
The primary Aggregated outcomes were pain over time,

patients in the MORE group

showed a 7\% reduction in pain, compared to a $3 \%$ increase in pain among the SG; thus pain intensity improved over the 8 weeks of MORE relative
Limitations

Participants were chronic pain patients that did not have to meet the criteria for OUD. Exploratory secondary analysis with relatively high attrition rates $(n=72 ; 63 \%$ of the randomly allocated

sample, $81 \%$ of those who attended one or more sessions) completed the treatment.
The population was not exclusively comprised of opioid misusers (67.3\% had COMM scores greater than or equal to 13). Specific response rate to the EMA was not reported. The 
Table 2 Characteristics of Studies Included in Systematic Review (Continued)

\begin{tabular}{|c|c|c|c|c|c|c|c|}
\hline Reference & $\begin{array}{l}\text { Treatment } \\
\text { condition }\end{array}$ & $\begin{array}{l}\text { Control } \\
\text { condition(s) }\end{array}$ & Overview of sample & $\begin{array}{l}\text { Data collection } \\
\text { time points }\end{array}$ & Outcome measures & Results & Limitations \\
\hline & & $\begin{array}{l}\text { ways of coping } \\
\text { with negative } \\
\text { emotions, the ef- } \\
\text { fect of life stress } \\
\text { on pain, the } \\
\text { stigma of opioid } \\
\text { use, risks of long- } \\
\text { term opioid use } \\
\text { and opioid de- } \\
\text { pendence, ac- } \\
\text { ceptance versus } \\
\text { denial, and plans } \\
\text { for the future. }\end{array}$ & & $\begin{array}{l}\text { each day. } \\
\text { Baseline and } \\
\text { post-treatment } \\
\text { assessment of } \\
\text { opioid misuse. }\end{array}$ & & $\begin{array}{l}\text { to the SG (an } \\
\text { estimated } \\
\text { improvement of } \\
0.7 \text { points on a } \\
\text { scale of 0-10). } \\
\text { Over the } 8 \\
\text { weeks of the } \\
\text { study } \\
\text { interventions, } \\
\text { participants in } \\
\text { MORE were } 2.75 \\
\text { times more } \\
\text { likely to be } \\
\text { affectively } \\
\text { regulated than } \\
\text { participants in } \\
\text { the SG. Mean } \\
\text { scores on the } \\
\text { COMM } \\
\text { decreased } \\
\text { during } \\
\text { treatment by } \\
7.06 \text { in the } \\
\text { MORE group } \\
\text { and by } 3.25 \text { in } \\
\text { SG. }\end{array}$ & $\begin{array}{l}\text { inclusionary } \\
\text { criteria required } \\
\text { completion of } \\
\text { at least one } \\
\text { EMA } \\
\text { measurement. }\end{array}$ \\
\hline $\begin{array}{l}\text { Garland } \\
\text { et al., } 2017 \\
\text { [49] }\end{array}$ & $\begin{array}{l}\text { MORE: } 8,120- \\
\text { min sessions. }\end{array}$ & $\begin{array}{l}\text { Support group } \\
\text { (SG): 8, 120- min } \\
\text { sessions. The } \\
\text { topics were } \\
\text { pertinent to } \\
\text { chronic pain and } \\
\text { long-term opioid } \\
\text { use and included: } \\
\text { the physical and } \\
\text { emotional aspects } \\
\text { of pain experi- } \\
\text { ence, ways of } \\
\text { coping with } \\
\text { chronic pain, } \\
\text { ways of coping } \\
\text { with negative } \\
\text { emotions, the ef- } \\
\text { fect of life stress } \\
\text { on pain, the } \\
\text { stigma of opioid } \\
\text { use, risks of long- } \\
\text { term opioid use } \\
\text { and opioid de- } \\
\text { pendence, ac- } \\
\text { ceptance versus } \\
\text { denial, and plans } \\
\text { for the future. }\end{array}$ & $\begin{array}{l}51 \text { chronic pain } \\
\text { patients (MORE } n= \\
\text { 20, SG } n=31 \text { ). } \\
\text { This subset was } \\
\text { taken from a larger } \\
\text { sample (115) during } \\
\text { an RCT testing the } \\
\text { effectiveness of the } \\
\text { MORE intervention } \\
\text { for individuals with } \\
\text { chronic pain. } 17 \\
\text { men and } 34 \\
\text { women, mean } \\
\text { age }=45.7 \text { (13.7) } \\
\text { years old. }\end{array}$ & $\begin{array}{l}\text { Baseline and } \\
\text { post-treatment. }\end{array}$ & $\begin{array}{l}\text { The primary } \\
\text { outcome was } \\
\text { changes in relative } \\
\text { responsiveness to } \\
\text { natural reward and } \\
\text { opioid cues from } \\
\text { pre- to post- } \\
\text { intervention. HR was } \\
\text { used to measure cue } \\
\text { responsiveness and } \\
\text { high- frequency } \\
\text { heart rate variability } \\
\text { (HRV) to index para- } \\
\text { sympathetic regula- } \\
\text { tion of hedonic } \\
\text { responses, including } \\
\text { attention to emo- } \\
\text { tional information. } \\
\text { Positive scores } \\
\text { indicated increased } \\
\text { natural reward cue- } \\
\text { elicited HR relative } \\
\text { to opioid cue- } \\
\text { elicited HR. Negative } \\
\text { scores indicated de- } \\
\text { creased cue-elicited } \\
\text { HR to natural reward } \\
\text { cues compared with } \\
\text { opioid cue-elicited } \\
\text { HR. }\end{array}$ & $\begin{array}{l}\text { There was a } \\
\text { significant } \\
\text { group } \times \text { time } \\
\text { effect on HRV } \\
\text { responsivity, } \\
\text { indicating that } \\
\text { compared to } \\
\text { the SG, the } \\
\text { MORE group } \\
\text { experienced } \\
\text { significantly } \\
\text { greater } \\
\text { increases in HRV } \\
\text { responsivity } \\
\text { during affective } \\
\text { picture viewing. } \\
\text { This would } \\
\text { indicate that } \\
\text { MORE may be } \\
\text { effective at } \\
\text { enhancing } \\
\text { autonomic } \\
\text { regulation of } \\
\text { stress created } \\
\text { by drug-related } \\
\text { stimuli. MORE } \\
\text { participants ex- } \\
\text { perienced } \\
\text { greater reduc- } \\
\text { tions in cue- } \\
\text { elicited HR. This } \\
\text { effect was most } \\
\text { prominent for } \\
\text { drug cue- } \\
\text { elicited HR. This } \\
\text { finding may } \\
\text { point to the } \\
\text { downregulation } \\
\text { of response to } \\
\text { drug-related } \\
\text { cues. }\end{array}$ & $\begin{array}{l}\text { Small sample } \\
\text { size. There } \\
\text { were only } 51 \\
\text { individuals (of } \\
\text { the larger 115) } \\
\text { who had } \\
\text { complete sets } \\
\text { of data for this } \\
\text { exploratory } \\
\text { hypothesis. } \\
\text { Participants } \\
\text { were chronic } \\
\text { pain patients } \\
\text { that did not } \\
\text { necessarily } \\
\text { meet the } \\
\text { criteria for } \\
\text { OUD. }\end{array}$ \\
\hline
\end{tabular}


Table 2 Characteristics of Studies Included in Systematic Review (Continued)

\begin{tabular}{|c|c|c|c|c|c|c|c|}
\hline Reference & $\begin{array}{l}\text { Treatment } \\
\text { condition }\end{array}$ & $\begin{array}{l}\text { Control } \\
\text { condition(s) }\end{array}$ & Overview of sample & $\begin{array}{l}\text { Data collection } \\
\text { time points }\end{array}$ & Outcome measures & Results & Limitations \\
\hline $\begin{array}{l}\text { Garland } \\
\text { et al., } 2019 \\
\text { [50] }\end{array}$ & $\begin{array}{l}\text { MORE: } 8,120- \\
\text { min sessions } \\
\text { held once a } \\
\text { week. }\end{array}$ & $\begin{array}{l}\text { Support Group } \\
\text { (SG): 8, 120- min } \\
\text { sessions held } \\
\text { once a week. } \\
\text { Included } \\
\text { discussions on } \\
\text { topics pertinent } \\
\text { to chronic pain } \\
\text { and long-term } \\
\text { opioid use includ- } \\
\text { ing: the lived ex- } \\
\text { perience of } \\
\text { chronic pain, } \\
\text { ways of coping } \\
\text { with chronic pain; } \\
\text { ways of coping } \\
\text { with negative } \\
\text { emotions, how } \\
\text { stressful life } \\
\text { events impact } \\
\text { pain, the stigma } \\
\text { of opioid use and } \\
\text { dependence, ad- } \\
\text { verse effects of } \\
\text { opioids, accept- } \\
\text { ance versus de- } \\
\text { nial, and plans for } \\
\text { the future. }\end{array}$ & $\begin{array}{l}95 \text { participants } \\
\text { recruited from } \\
\text { primary care and } \\
\text { pain clinics in Salt } \\
\text { Lake City, Utah. } 56.8 \\
\text { (11.7) years old, 66\% } \\
\text { female, } 89 \% \text { non- } \\
\text { Hispanic White, } \\
122.87 \text { (93.6) } \\
\text { months of opioid } \\
\text { use. }\end{array}$ & $\begin{array}{l}\text { Baseline, post- } \\
\text { intervention, } \\
\text { and 3- month } \\
\text { follow-up. }\end{array}$ & $\begin{array}{l}\text { Primary outcomes } \\
\text { were: positive affect } \\
\text { (PANAS), meaning in } \\
\text { life (MLQ), savoring } \\
\text { (MSS), self- } \\
\text { transcendence } \\
\text { (NADA), pain severity } \\
\text { (BPI), and opioid } \\
\text { misuse risk (COMM). }\end{array}$ & $\begin{array}{l}\text { There was a } \\
\text { significant effect } \\
\text { of intervention } \\
\text { group on } \\
\text { change in } \\
\text { positive affect, } \\
\text { meaning in life, } \\
\text { savoring, and } \\
\text { self- } \\
\text { transcendence, } \\
\text { indicating that } \\
\text { MORE increased } \\
\text { these attributes } \\
\text { to a greater ex- } \\
\text { tent than did } \\
\text { the SG. For the } \\
\text { clinical variables, } \\
\text { there was a sig- } \\
\text { nificant effect of } \\
\text { intervention } \\
\text { group on resi- } \\
\text { dualized change } \\
\text { in pain at post- } \\
\text { treatment and } \\
\text { change in opi- } \\
\text { oid misuse risk } \\
\text { by 3-month } \\
\text { follow-up, such } \\
\text { that MORE re- } \\
\text { sulted in greater } \\
\text { improvements } \\
\text { in these vari- } \\
\text { ables than did } \\
\text { the SG. }\end{array}$ & $\begin{array}{l}\text { The } \\
\text { participants } \\
\text { were opioid- } \\
\text { treated chronic } \\
\text { pain patients } \\
\text { and did not } \\
\text { necessarily } \\
\text { meet the cri- } \\
\text { teria for OUD. } \\
\text { Attrition rates } \\
\text { were high } \\
(47.4 \%), \text { but } \\
\text { comparable to } \\
\text { that of other } \\
\text { opioid } \\
\text { interventions. }\end{array}$ \\
\hline $\begin{array}{l}\text { Garland } \\
\text { et al., } 2019 \\
\text { [51] }\end{array}$ & $\begin{array}{l}\text { MORE + MMT: } \\
8,120-\mathrm{min} \\
\text { sessions. }\end{array}$ & $\begin{array}{l}\text { TAU + MMT: 8, } \\
\text { 120-min sessions. } \\
\text { TAU consisted of } \\
\text { individual and } \\
\text { group therapy } \\
\text { provided by par- } \\
\text { ticipating in MMT } \\
\text { treatment agen- } \\
\text { cies. TAU thera- } \\
\text { peutic ap- } \\
\text { proaches in- } \\
\text { cluded process- } \\
\text { oriented, present- } \\
\text { centered therapy, } \\
\text { and cognitive- } \\
\text { behavioral coping } \\
\text { skills training but } \\
\text { did not include } \\
\text { formal } \\
\text { mindfulness- } \\
\text { based } \\
\text { intervention. }\end{array}$ & $\begin{array}{l}30 \text { individuals } \\
\text { enrolled in an MMT } \\
\text { program (MORE = } \\
15 ; \text { TAU = 15). } 50.4 \\
\text { (8.44) years old, } 50 \% \\
\text { female, } 36.6 \% \text { non- } \\
\text { Hispanic White, } 67 \% \\
\text { reported using } \\
\text { heroin in the past } \\
30 \text { days, and had } \\
\text { received a median } \\
\text { of } 7 \text { months of MMT } \\
\text { (range < } 1 \text { month to } \\
35 \text { years). }\end{array}$ & $\begin{array}{l}112 \text { EMA } \\
\text { collection } \\
\text { points (2 } \\
\text { assessments } \\
\text { per day/ } 56 \\
\text { days). }\end{array}$ & $\begin{array}{l}\text { Primary outcomes } \\
\text { were craving and } \\
\text { urge to use opioids, } \\
\text { pain intensity, and } \\
\text { affective state. The } \\
\text { secondary outcome } \\
\text { was event } \\
\text { contingent craving. }\end{array}$ & $\begin{array}{l}\text { MORE } \\
\text { participants } \\
\text { reported greater } \\
\text { decreases in } \\
\text { several } \\
\text { categories } \\
\text { including: } \\
\text { opioid wanting } \\
\text { (44\% decrease), } \\
\text { opioid urge } \\
\text { (50\% decrease), } \\
\text { pain } \\
\text { unpleasantness } \\
\text { (13\% decrease), } \\
\text { and stress (26\% } \\
\text { decrease). } \\
\text { Event- } \\
\text { contingent EMA } \\
\text { showed MORE } \\
\text { participants to } \\
\text { report a greater } \\
\text { number of crav- } \\
\text { ings ( } n=303 \text { ) } \\
\text { than partici- } \\
\text { pants in TAU } \\
\text { ( } n=87) \text {. Yet, } \\
\text { those cravings } \\
\text { were reported } \\
\text { as being signifi- } \\
\text { cantly less in- } \\
\text { tense than } \\
\text { those experi- } \\
\text { enced by }\end{array}$ & $\begin{array}{l}\text { The daily } \\
\text { response rate } \\
\text { to random } \\
\text { EMA probes } \\
\text { over the two } \\
\text { months of the } \\
\text { intervention } \\
\text { was } 62 \% \text {. }\end{array}$ \\
\hline
\end{tabular}


Table 2 Characteristics of Studies Included in Systematic Review (Continued)

\begin{tabular}{|c|c|c|c|c|c|c|c|}
\hline Reference & $\begin{array}{l}\text { Treatment } \\
\text { condition }\end{array}$ & $\begin{array}{l}\text { Control } \\
\text { condition(s) }\end{array}$ & Overview of sample & $\begin{array}{l}\text { Data collection } \\
\text { time points }\end{array}$ & Outcome measures & Results & Limitations \\
\hline & & & & & & $\begin{array}{l}\text { participants in } \\
\text { TAU. Participants } \\
\text { in MORE had } \\
68 \% \text { less severe } \\
\text { opioid wanting } \\
\text { and } 56 \% \text { weaker } \\
\text { opioid urges } \\
\text { than partici- } \\
\text { pants in TAU. }\end{array}$ & \\
\hline $\begin{array}{l}\text { Garland } \\
\text { et al., } 2019 \\
\text { [52] }\end{array}$ & $\begin{array}{l}\text { MORE: } 8,120- \\
\text { min sessions. }\end{array}$ & $\begin{array}{l}\text { Active Support } \\
\text { Group (SG): } 8 \text {, } \\
\text { 120-min. Client- } \\
\text { centered group } \\
\text { format that facili- } \\
\text { tated emotional } \\
\text { expression and } \\
\text { discussion of } \\
\text { topics pertinent } \\
\text { to chronic pain } \\
\text { and opioid use/ } \\
\text { misuse. }\end{array}$ & $\begin{array}{l}135 \text { individuals } \\
\text { recruited from } \\
\text { primary care and } \\
\text { pain clinics in Salt } \\
\text { Lake City, UT } \\
\text { (Sample } 1=40 \text {, } \\
\text { Sample } 2=31 \text {, } \\
\text { Sample } 3=64) .55 .4 \\
(11.1), 57.8(11.3) \text {, } \\
56.7(10.9) \text { years old } \\
\text { respectively per } \\
\text { sample. } \\
57.5,12.9 \text {, and } 65.6 \% \\
\text { female. 90, 80.6, and } \\
82.8 \% \text { non-Hispanic } \\
\text { White. } \\
\text { Average duration of } \\
\text { opioid use, } 10.13 \\
\text { (7.17) years }\end{array}$ & $\begin{array}{l}\text { Baseline and } \\
\text { post- } \\
\text { intervention. }\end{array}$ & $\begin{array}{l}\text { Experiment } 1 \\
\text { assessed the effects } \\
\text { of treatment on LPP } \\
\text { indices of opioid } \\
\text { cue-reactivity relative } \\
\text { to reactivity to neu- } \\
\text { tral cues. } \\
\text { Experiment } 2 \\
\text { assessed the effects } \\
\text { of treatment on the } \\
\text { capacity to down- } \\
\text { regulate the LPP re- } \\
\text { sponse to opioid } \\
\text { cues. Experiment } 3 \\
\text { assessed the effects } \\
\text { of treatment on the } \\
\text { capacity to up- } \\
\text { regulate the LPP re- } \\
\text { sponse to natural re- } \\
\text { ward cues. } \\
\text { Experiment } 4 \text { evalu- } \\
\text { ated affect ratings in } \\
\text { response to natural } \\
\text { reward cues col- } \\
\text { lected from a sample } \\
\text { of opioid-treated } \\
\text { chronic pain patients } \\
\text { participating in a } \\
\text { stage } 2 \text { RCT of } \\
\text { MORE. }\end{array}$ & $\begin{array}{l}\text { For experiment } \\
\text { 1, within-group } \\
\text { comparisons in- } \\
\text { dicated that SG } \\
\text { participants' } \\
\text { post-treatment } \\
\text { LPP activations } \\
\text { remained higher } \\
\text { in response to } \\
\text { opioid cues } \\
\text { compared to } \\
\text { the neutral cues, } \\
\text { suggesting an } \\
\text { opioid cue- } \\
\text { reactivity. Rela- } \\
\text { tive to those in } \\
\text { the SG, partici- } \\
\text { pants who were } \\
\text { treated with } \\
\text { MORE exhibited } \\
\text { significantly } \\
\text { greater de- } \\
\text { creases in the } \\
\text { LPP response to } \\
\text { opioid cues dur- } \\
\text { ing regulation } \\
\text { (regulate < } \\
\text { view) from pre- } \\
\text { treatment to } \\
\text { post-treatment. } \\
\text { MORE partici- } \\
\text { pants showed } \\
\text { significantly } \\
\text { greater in- } \\
\text { creases in LPP } \\
\text { response to nat- } \\
\text { ural reward cues } \\
\text { during regula- } \\
\text { tion from pre- } \\
\text { treatment to } \\
\text { post-treatment. } \\
\text { Relative to those } \\
\text { in the SG, partic- } \\
\text { ipants who } \\
\text { were treated } \\
\text { with MORE ex- } \\
\text { hibited signifi- } \\
\text { cantly greater } \\
\text { positive affective } \\
\text { responses to } \\
\text { natural reward } \\
\text { cues from pre- } \\
\text { treatment to } \\
\text { post-treatment. }\end{array}$ & $\begin{array}{l}\text { The study was } \\
\text { not a clinical } \\
\text { trial powered } \\
\text { to detect } \\
\text { changes in } \\
\text { treatment } \\
\text { outcomes. }\end{array}$ \\
\hline
\end{tabular}

\footnotetext{
Price et al. MABT: 8, 902019 [23] min sessions.

TAU: Group sessions $2-3 \times$ /
}

Women $(\mathrm{MABT}=93$ initially, 74 included
Baseline and 3- Substance use month post- outcomes were $\%$ of received MABT
Only examined short- term 
Table 2 Characteristics of Studies Included in Systematic Review (Continued)

\begin{tabular}{|c|c|c|c|c|c|c|c|}
\hline Reference & $\begin{array}{l}\text { Treatment } \\
\text { condition }\end{array}$ & $\begin{array}{l}\text { Control } \\
\text { condition(s) }\end{array}$ & Overview of sample & $\begin{array}{l}\text { Data collection } \\
\text { time points }\end{array}$ & Outcome measures & Results & Limitations \\
\hline & & $\begin{array}{l}\text { week for 10-14 } \\
\text { weeks, individual } \\
\text { counseling at } \\
\text { least } 1 \times / \text { month. } \\
\text { Included } \\
\text { education about } \\
\text { alcohol and drug } \\
\text { use and } \\
\text { participation in } \\
\text { self-help groups } \\
\text { (e.g., } 12 \text {-step) for } \\
\text { SUD. Women's } \\
\text { health education } \\
\text { (WHE): } 8,90-\text {-min } \\
\text { group sessions. } \\
\text { Included topics } \\
\text { such as under- } \\
\text { standing the fe- } \\
\text { male body, } \\
\text { reproductive } \\
\text { health, cardiovas- } \\
\text { cular health, and } \\
\text { sexually transmit- } \\
\text { ted diseases. }\end{array}$ & $\begin{array}{l}\text { in analyses; WHE = } \\
56 \text { initially, } 46 \\
\text { included in analyses; } \\
\text { TAU = } 68 \text { initially, } 67 \\
\text { included in } \\
\text { analyses) recruited } \\
\text { from outpatient } \\
\text { SUD treatment } \\
\text { programs who were } \\
\text { currently enrolled in } \\
\text { intensive outpatient } \\
\text { treatment. Median } \\
\text { age of } 35 \text { years, } 75 \% \\
\text { non-Hispanic white. }\end{array}$ & $\begin{array}{l}\text { baseline } \\
\text { follow-up }\end{array}$ & $\begin{array}{l}\text { days abstinent and a } \\
\text { binary indicator of } \\
\text { relapse (TLFB, urine } \\
\text { drug screen, } \\
\text { electronic health } \\
\text { records) and craving } \\
\text { (PACS). Mindfulness } \\
\text { outcomes were } \\
\text { interoceptive } \\
\text { awareness (MAIA) } \\
\text { and mindfulness } \\
\text { (FIM). Psychiatric } \\
\text { distress outcomes } \\
\text { were DERS, } \\
\text { psychophysiological } \\
\text { response to a } \\
\text { stressful video, } \\
\text { rumination, and } \\
\text { body awareness } \\
\text { (Respiratory Sinus } \\
\text { Arrhythmia; RSA), } \\
\text { BDI-II, and PSS-SR. } \\
\text { Also assessed patient } \\
\text { satisfaction. }\end{array}$ & $\begin{array}{l}\text { or WHE, } \\
\text { compared with } \\
\text { TAU, showed } \\
\text { significantly } \\
\text { greater } \\
\text { improvement in } \\
\text { the proportion } \\
\text { of days } \\
\text { abstinent; } \\
\text { however, there } \\
\text { were no } \\
\text { significant } \\
\text { differences } \\
\text { across groups in } \\
\text { relapse or } \\
\text { craving. MABT } \\
\text { produced } \\
\text { significantly } \\
\text { greater } \\
\text { improvements } \\
\text { in mindfulness, } \\
\text { as compared to } \\
\text { the control } \\
\text { conditions, in } \\
\text { analyses } \\
\text { including } \\
\text { individuals who } \\
\text { attended 6+ } \\
\text { groups, but not } \\
\text { in intent to treat } \\
\text { (ITT) analyses. } \\
\text { MABT was } \\
\text { associated with } \\
\text { greater } \\
\text { improvements } \\
\text { in } 6 / 8 \text { MAIA } \\
\text { subscales, as } \\
\text { compared to } \\
\text { the control } \\
\text { conditions. } \\
\text { MABT was } \\
\text { associated with } \\
\text { improvements } \\
\text { in DERS scores } \\
\text { and RSA during } \\
\text { the film } \\
\text { reactivity task, } \\
\text { the body } \\
\text { awareness } \\
\text { reactivity task, } \\
\text { and (in analyses } \\
\text { of those who } \\
\text { completed 6+ } \\
\text { groups) resting } \\
\text { RSA, as } \\
\text { compared to } \\
\text { the control } \\
\text { conditions. As } \\
\text { compared to } \\
\text { control } \\
\text { conditions, } \\
\text { MABT was } \\
\text { associated with } \\
\text { greater } \\
\text { improvements } \\
\text { in BDI-II scores } \\
\text { among those }\end{array}$ & $\begin{array}{l}\text { follow-up. } \\
\text { Findings might } \\
\text { not generalize } \\
\text { to men. }\end{array}$ \\
\hline
\end{tabular}


Table 2 Characteristics of Studies Included in Systematic Review (Continued)

\begin{tabular}{|c|c|c|c|c|c|c|c|}
\hline Reference & $\begin{array}{l}\text { Treatment } \\
\text { condition }\end{array}$ & $\begin{array}{l}\text { Control } \\
\text { condition(s) }\end{array}$ & Overview of sample & $\begin{array}{l}\text { Data collection } \\
\text { time points }\end{array}$ & Outcome measures & Results & Limitations \\
\hline & & & & & & $\begin{array}{l}\text { who completed } \\
6+\text { groups, but } \\
\text { was not associ- } \\
\text { ated with im- } \\
\text { provements in } \\
\text { BDI-II or PSS- SR } \\
\text { scores in ITT } \\
\text { analyses. } 72 \% \text { of } \\
\text { MABT and 63\% } \\
\text { of WHE partici- } \\
\text { pants endorsed } \\
\text { highly positive } \\
\text { treatment satis- } \\
\text { faction ratings } \\
\text { of "very much" } \\
\text { or "extremely." }\end{array}$ & \\
\hline $\begin{array}{l}\text { Price et al. } \\
2019 \text { [53] }\end{array}$ & $\begin{array}{l}\text { MABT: } 8,90- \\
\text { min sessions. }\end{array}$ & $\begin{array}{l}\text { TAU: Group } \\
\text { sessions 2-3x/ } \\
\text { week for 10-14 } \\
\text { weeks, individual } \\
\text { counseling at } \\
\text { least 1x/month. } \\
\text { Included educa- } \\
\text { tion about alco- } \\
\text { hol and drug use } \\
\text { and participation } \\
\text { in self-help } \\
\text { groups (e.g., 12- } \\
\text { step) for SUD. } \\
\text { Women's health } \\
\text { education (WHE): } \\
\text { 8, 90-min group } \\
\text { sessions. }\end{array}$ & Reported above. & $\begin{array}{l}\text { Baseline, 3-, 6-, } \\
\text { and 12-month } \\
\text { follow-up. }\end{array}$ & $\begin{array}{l}\text { The primary } \\
\text { outcome was days } \\
\text { abstinent from } \\
\text { substance use (TLFB). } \\
\text { Secondary outcomes } \\
\text { included measures } \\
\text { of emotion } \\
\text { regulation (DERS), } \\
\text { craving (PACS), } \\
\text { psychological } \\
\text { distress (PSS-SR and } \\
\text { BDI-II), mindfulness } \\
\text { (FMI), and interocep- } \\
\text { tive awareness } \\
\text { (MAIA), respiratory } \\
\text { sinus arrhythmia } \\
\text { (Tonic RSA). }\end{array}$ & $\begin{array}{l}\text { Primary } \\
\text { Outcome: The } \\
\text { ID sample } \\
\text { showed a } \\
\text { significant } \\
\text { difference } \\
\text { between } \\
\text { groups. MABT \& } \\
\text { WHE PDA > TAU } \\
\text { at } 6 \text { months, } \\
\text { MABT > TAU at } \\
12 \text { months. } \\
\text { MABT } \\
\text { improvements } \\
\text { maintained the } \\
\text { increased level } \\
\text { while WHE \& } \\
\text { TAU decreased } \\
\text { over time. No } \\
\text { differences } \\
\text { between MABT } \\
\text { and WHE at any } \\
\text { time point. } \\
\text { Secondary } \\
\text { Outcomes: Self- } \\
\text { report emotion } \\
\text { dysregulation } \\
\text { had no signifi- } \\
\text { cant difference } \\
\text { at any time } \\
\text { point in ID and } \\
\text { ITT analysis. } \\
\text { Craving: overall } \\
\text { ITT \& ID showed } \\
\text { significant im- } \\
\text { provements at } \\
6-\text { and } 12- \\
\text { months. Psycho- } \\
\text { logical distress. } \\
\text { No longitudinal } \\
\text { difference in } \\
\text { mindfulness. } \\
\text { Overall signifi- } \\
\text { cant difference } \\
\text { in ID sample } \\
\text { with increases } \\
\text { in mindfulness } \\
\text { skills at 3- and } \\
6 \text {-months. In- } \\
\text { teroceptive } \\
\text { awareness. }\end{array}$ & $\begin{array}{l}\text { Low } \\
\text { generalizability } \\
\text { due to the low } \\
\text { SES of } \\
\text { participants, } \\
\text { and the fact } \\
\text { that all } \\
\text { members were } \\
\text { women. Study } \\
\text { is re-reporting } \\
\text { some results } \\
\text { with an added } \\
\text { time point. }\end{array}$ \\
\hline
\end{tabular}


Table 2 Characteristics of Studies Included in Systematic Review (Continued)

\begin{tabular}{|c|c|c|c|}
\hline Reference & $\begin{array}{l}\text { Treatment } \\
\text { condition }\end{array}$ & $\begin{array}{l}\text { Control } \\
\text { condition(s) }\end{array}$ & Overview of sample \\
\hline \multicolumn{4}{|c|}{ Mindfulness-Based Addiction Treatment (MBAT) } \\
\hline $\begin{array}{l}\text { Spears } \\
\text { et al., } 2017 \\
{[26]}\end{array}$ & $\begin{array}{l}\text { MBAT: } 8,120- \\
\text { min group } \\
\text { sessions. }\end{array}$ & $\begin{array}{l}\text { CBT (Manualized): } \\
\text { 8, 120- min group } \\
\text { session treatment } \\
\text { for smoking } \\
\text { cessation. Quit } \\
\text { day scheduled for } \\
\text { Session 5. UC: 4, } \\
\text { 5-10 min } \\
\text { counseling } \\
\text { sessions. Intended } \\
\text { to be equivalent } \\
\text { to typical support } \\
\text { from a health } \\
\text { care provider. } \\
\text { Content } \\
\text { emphasized } \\
\text { problem solving } \\
\text { and coping skills } \\
\text { training. Quit day } \\
\text { scheduled for } \\
\text { Session } 3 \text {. }\end{array}$ & $\begin{array}{l}412 \text { adults who } \\
\text { were current } \\
\text { smokers (average } \\
5+\text { cigarettes per } \\
\text { day for the past } \\
\text { year), motivated to } \\
\text { quit within } 30 \text { days. } \\
54.9 \% \text { female, } 48.2 \% \\
\text { African-American, } \\
41.5 \% \text { non-Latino } \\
\text { white. Mean age } \\
\text { not reported. }\end{array}$ \\
\hline
\end{tabular}

\begin{tabular}{|c|c|c|c|}
\hline $\begin{array}{l}\text { Vidrine } \\
\text { et al., } 2016 \\
\text { [25] }\end{array}$ & $\begin{array}{l}\text { MBAT: } 8,120- \\
\text { min group } \\
\text { sessions. }\end{array}$ & $\begin{array}{l}\text { CBT (Manualized): } \\
8,120-\text { min group } \\
\text { session treatment } \\
\text { for smoking ces- } \\
\text { sation. Quit day } \\
\text { scheduled for } \\
\text { Session } 5 \text {. } \\
\text { UC: } 4,5-10 \text { min } \\
\text { counseling } \\
\text { sessions. Intended } \\
\text { to be equivalent } \\
\text { to typical support } \\
\text { from a health } \\
\text { care provider. } \\
\text { Content } \\
\text { emphasized } \\
\text { problem solving } \\
\text { and coping skills } \\
\text { training. Quit day } \\
\text { scheduled for } \\
\text { sccinn? }\end{array}$ & $\begin{array}{l}412 \text { adults, current } \\
\text { smokers (average } \\
5+\text { cigarettes per } \\
\text { day for the past } \\
\text { year), motivated to } \\
\text { quit within } 30 \text { days. } \\
54.9 \% \text { female, } 48.2 \% \\
\text { African-American, } \\
41.5 \% \text { non-Latino } \\
\text { white. Mean age } \\
\text { not reported. }\end{array}$ \\
\hline
\end{tabular}

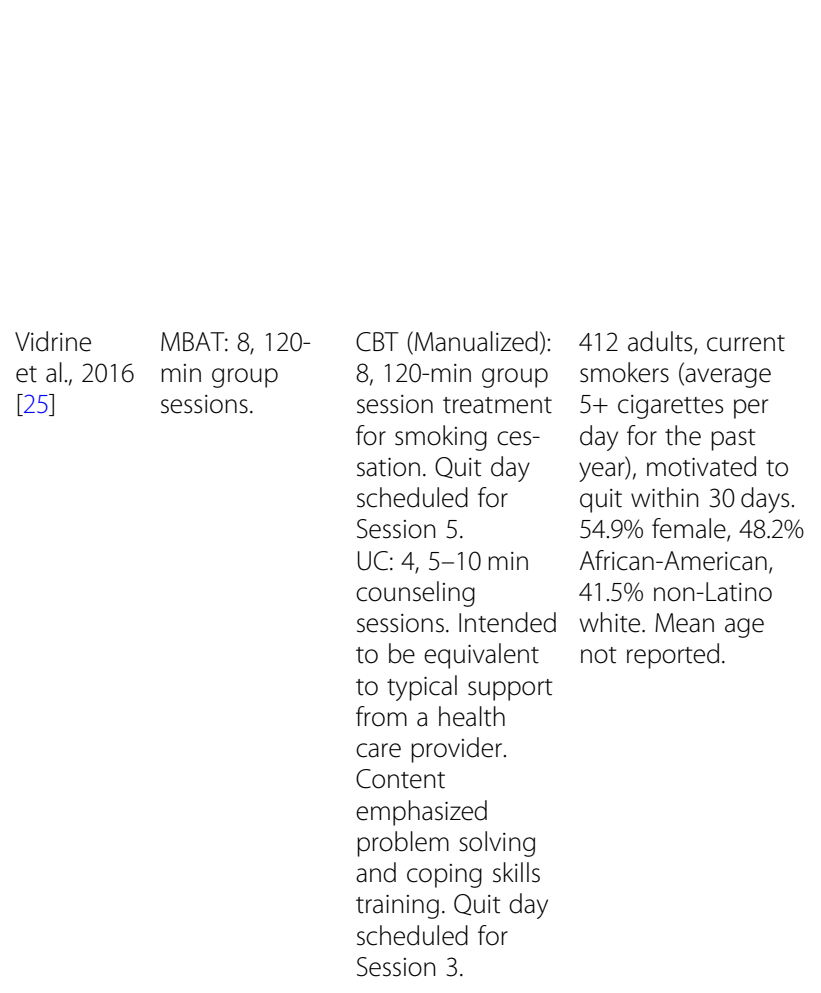

Data collection time points

Baseline,
treatment
weeks 3-8, 26-
week follow-up
treatment, 26week follow-up

Nicotine

dependence (HSI),

self-report average

days using mindfulness techniques, and smoking abstinence was biochemically confirmed.

Smoking cessation
outcomes were:
nicotine

Participants in the MBAT condition perceived smoking dependence and greater volitional moking withdrawal control over smoking and lower volatility of anger than (SES), and at- CBT and UC. No other significant differences nor (PANAS), indirect effects toms (CES D), Participants in tress (PSS), and the MBAT condition reported lower anxiety, attentional bias, concentration difficulties, craving, smoking dependence motives and higher selfefficacy for negative affect relative to UC.

\section{MBAT}

participants reported the highest percentage of 7-day point prevalence abstinence, but not a significant difference. Among participants classified as smoking at final tx sessions, recovery of abstinence was examined. MBAT condition had significantly higher recovery than CBT

(Cohen's $d=.88$ ) and UC

(Cohen's $d=.79$ )
There was a heavy reliance on repeated administration of self- report questionnaires with significant time lapses between assessments.
The same therapists delivered all treatments. No assessment of fidelity 
Table 2 Characteristics of Studies Included in Systematic Review (Continued)

\begin{tabular}{|c|c|c|c|c|}
\hline Reference & $\begin{array}{l}\text { Treatment } \\
\text { condition }\end{array}$ & $\begin{array}{l}\text { Control } \\
\text { condition(s) }\end{array}$ & Overview of sample & $\begin{array}{l}\text { Data collection } \\
\text { time points }\end{array}$ \\
\hline $\begin{array}{l}\text { et al. } 2017 \\
\text { [16] }\end{array}$ & $\begin{array}{l}\text { sessions over } 4 \\
\text { weeks. }\end{array}$ & $\begin{array}{l}\text { Smoking (FFS): } 8 \text {, } \\
90-\text { min sessions } \\
\text { over } 4 \text { weeks. } \\
\text { Topics included } \\
\text { cognitive strat- } \\
\text { egies for coping } \\
\text { with cravings and } \\
\text { stress/negative } \\
\text { emotions, behav- } \\
\text { ior modification, } \\
\text { and relapse } \\
\text { prevention. }\end{array}$ & $\begin{array}{l}\text { FFS }=12 \text { ) who } \\
\text { smoked }>10 \\
\text { cigarettes/day, had } \\
\text { fewer than } 3 \\
\text { months of } \\
\text { abstinence in the } \\
\text { previous year, and } \\
\text { were interested in } \\
\text { smoking cessation. } \\
\text { All participants were } \\
\text { part of a larger trial } \\
\text { comparing MT and } \\
\text { FFS and participants } \\
\text { included in the } \\
\text { present analysis } \\
\text { represent a subset } \\
\text { who completed a } \\
\text { neuroimaging task. } \\
69.6 \% \text { male, } 48.3 \\
\text { (7.0) years old, } \\
60.9 \% \text { non-Hispanic } \\
\text { white. }\end{array}$ & $\begin{array}{l}\text { treatment, and } \\
\text { 3-month post- } \\
\text { treatment } \\
\text { follow-up. }\end{array}$ \\
\hline
\end{tabular}

Moment-by-Moment in Women's Recovery (MMWR)

$\begin{array}{lll}\text { Black et al., } & \text { Residential SUD } & \text { Neurobiology of } \\ 2019[17] & \text { treatment } & \text { addiction (NA): } \\ & \text { program + } & \text { 12, 80-min group } \\ & \text { MMWR: 12,80- } & \text { sessions delivered } \\ & \text { min sessions } & \text { twice weekly. } \\ & \text { delivered twice } & \text { Topics included: } \\ & \text { weekly. MMWR } & \text { definition of }\end{array}$

200 female patients (MMWR- 100, NA-

100) at a residential

SUD treatment facility. $32.5(9.1)$ years old, $21.0 \%$ non- Hispanic White, $46.5 \%$
Baseline and postintervention assessment of the self-report measures. 150day cap

Outcome measures

Results

conditions

were reductions in

average cigarettes/

day from pre- to

post-treatment

(TLFB).

Also examined fMRI

and self-reported

stress reactivity and

craving to stress/

negative scrips and

neutral/relaxing

scripts.

reduced

smoking

(cigarettes/day),

but the MT

group showed

greater

reductions

during

treatment and

at the 3-month

post-treatment

follow-up. The

MT and FFS

groups did not

differ in their

self-reported

stress or craving

to stressful/

negative scripts

at the post-

treatment fMR

session and

there were no

significant

group differ-

ences in brain

activity during

neutral scenar-

ios. Participants

in the FFS

group (vs. MT)

exhibited in-

creased neural

reactivity in sev-

eral brain re-

gions during

the stressful

scripts. The MT

group did not

demonstrate

greater neural

reactivity in any

region. Lower

reactivity in sev-

eral brain re-

gions during

stressful scripts

in the MT con-

dition (vs. the

FFS condition)

was associated

with a greater

reduction in

smoking after

treatment and

at 3-month

follow-up.

The primary

outcome was

discharge status and

days until discharge.

Secondary outcomes

were self-report

measure scores of
The hazard ratio It is possible

for retention

that the

control

curriculum was

to-large effect

size, suggesting equally

the clinical rele- beneficial to

vance of adding some self- size. Only

fore could

ductions in

neural stress

reactivity. 
Table 2 Characteristics of Studies Included in Systematic Review (Continued)

\begin{tabular}{|c|c|c|c|c|c|c|c|}
\hline Reference & $\begin{array}{l}\text { Treatment } \\
\text { condition }\end{array}$ & $\begin{array}{l}\text { Control } \\
\text { condition(s) }\end{array}$ & Overview of sample & $\begin{array}{l}\text { Data collection } \\
\text { time points }\end{array}$ & Outcome measures & Results & Limitations \\
\hline & $\begin{array}{l}\text { was delivered } \\
\text { across } 6 \text { weeks } \\
\text { (as opposed to } \\
\text { the standard } \\
\text { 12) to better fit } \\
\text { the residential } \\
\text { site clinical } \\
\text { services } \\
\text { structure. The } \\
\text { residential } \\
\text { program } \\
\text { included: } \\
\text { mental health } \\
\text { and SUD } \\
\text { diagnosis and } \\
\text { treatment } \\
\text { (individual and } \\
\text { group), trauma } \\
\text { education and } \\
\text { support, } \\
\text { vocational } \\
\text { training, } \\
\text { nutritional } \\
\text { education and } \\
\text { support, health } \\
\text { and wellness } \\
\text { activities, and } \\
12 \text {-step meet- } \\
\text { ings, among } \\
\text { other services. }\end{array}$ & $\begin{array}{l}\text { addiction and } \\
\text { why it is a brain } \\
\text { disease; brain } \\
\text { structures and } \\
\text { functions and } \\
\text { those related to } \\
\text { addiction; effects } \\
\text { of various types } \\
\text { of substances on } \\
\text { the brain; reward- } \\
\text { ing effects of sub- } \\
\text { stances and how } \\
\text { these effects lead } \\
\text { to addiction; defi- } \\
\text { nitions and brain } \\
\text { functions related } \\
\text { to craving and } \\
\text { withdrawal; and } \\
\text { the roles of treat- } \\
\text { ment in recovery. }\end{array}$ & $\begin{array}{l}\text { had less than a high } \\
\text { school education, } \\
62 \% \text { had been in- } \\
\text { carcerated in the } 8 \\
\text { months prior to resi- } \\
\text { dential treatment } \\
\text { entry, and } 76 \% \text { were } \\
\text { amphetamine/ } \\
\text { methamphetamine- } \\
\text { mine users }\end{array}$ & $\begin{array}{l}\text { (beginning on } \\
\text { the first day of } \\
\text { the interven- } \\
\text { tion) for dis- } \\
\text { charge status } \\
\text { (completer, } \\
\text { non- com- } \\
\text { pleter with sat- } \\
\text { isfactory pro- } \\
\text { gress, non- } \\
\text { completer } \\
\text { without satis- } \\
\text { factory pro- } \\
\text { gress, in- } \\
\text { residence). }\end{array}$ & $\begin{array}{l}\text { study intervention } \\
\text { mechanisms of ac- } \\
\text { tion: mindfulness } \\
\text { (FFMQ-SF), perceived } \\
\text { stress (PSS-10), dis- } \\
\text { tress tolerance and } \\
\text { emotion regulation } \\
\text { (DERS), distress } \\
\text { (DASS-21), affect } \\
\text { (PANAS), and drug } \\
\text { and alcohol craving } \\
\text { (PACS). }\end{array}$ & $\begin{array}{l}\text { MMWR to an } \\
\text { all-women's, } \\
\text { ethno-racially di- } \\
\text { verse, SUD resi- } \\
\text { dential treat- } \\
\text { ment center. } \\
\text { The length of } \\
\text { stay in residen- } \\
\text { tial treatment } \\
\text { from study } \\
\text { intervention } \\
\text { start date to } \\
\text { analytic end- } \\
\text { point (150 days) } \\
\text { was } 94.4 \text { days. } \\
\text { There were } 74 \\
\text { treatment com- } \\
\text { pleters (43/100 } \\
\text { in MMWR, } 31 / \\
100 \text { in the NA } \\
\text { group), } 42 \\
\text { women still in } \\
\text { residence (15/ } \\
100 \text { in MMWR, } \\
27 / 100 \text { in the } \\
\text { NA group). } \\
\text { There were } 84 \\
\text { treatment non- } \\
\text { completers, but } \\
\text { satisfactory pro- } \\
\text { gress was made } \\
\text { by } 16 / 42 \\
\text { women in the } \\
\text { MMWR group } \\
\text { and } 10 / 42 \\
\text { women in the } \\
\text { NA group. For } \\
\text { the ITT, risk of } \\
\text { non-completion } \\
\text { without im- } \\
\text { provement was } \\
\text { lower in MMWR } \\
\text { as compared to } \\
\text { the NA group } \\
\text { after the study } \\
\text { intervention. At } \\
\text { post- } \\
\text { intervention, all } \\
\text { eight secondary } \\
\text { outcome mea-- } \\
\text { surements had } \\
\text { improved for } \\
\text { both groups. }\end{array}$ & $\begin{array}{l}\text { reported mea- } \\
\text { sures of thera- } \\
\text { peutic change, } \\
\text { which was evi- } \\
\text { dent for signifi- } \\
\text { cantly im- } \\
\text { proved scores } \\
\text { in both groups } \\
\text { for the FFMQ, } \\
\text { DTS, DERS, and } \\
\text { PACS. }\end{array}$ \\
\hline
\end{tabular}

conjunction with the Brazilian Ministry of Health's tobacco cessation standard treatment (ST) protocol to the stand-alone ST protocol [41]. The intention to treat (ITT) analysis showed that at week 24 abstinence was $14.3 \%$ for ST, but $20.1 \%$ for MBRP, indicating that continued smoking rates were $37 \%$ lower in the MBRP group. The secondary outcomes showed that individuals in the MBRP group had reductions in craving based on the Questionnaire of Smoking Urges (QSU) from week 4 to week $12($ Mean $=17.58)$ and increases in levels of mindfulness based on the Five Facet Mindfulness Questionnaire-Brazil (FFMQ-BR) (Mean = -7.83).

Three recent studies have also demonstrated initial efficacy of MBRP among additional international samples, including those with opioid use disorder and cooccurring depression. Yaghubi and colleagues compared the efficacy of MBRP relative to TAU among male, opioid-dependent patients referred for methadone maintenance treatment in Iran [43]. MBRP participants experienced higher rates of abstinence at post-treatment and 
at two-month follow-up relative to TAU participants. Impulsivity scores among MBRP participants decreased significantly over time relative to TAU. In addition, an eight-week intervention comparing MBRP to treatment as usual for Iranian males in methadone maintenance treatment $(n=60)$ was conducted by Abed \& Shahidi [30]. Participants in the MBRP condition reported significant decreases in craving and desire to use. Lower levels of positive urinalysis tests were reported among MBRP participants over the three-month follow-up; however, significance testing was not conducted. Zemestani \& Ottaviani compared the efficacy of MBRP relative to TAU among substance dependent (DSM-IV criteria), moderately depressed Iranian adults receiving outpatient treatment for substance dependence [44]. MBRP participants reported decreased depression, anxiety, and craving relative to TAU participants.

Recent trials have also focused on adapting MBRP to improve implementation and efficacy. In an effort to make MBRP implementation more practical, Shorey and colleagues compared blended MBRP and acceptance and commitment therapy (ACT) to TAU among adult, residential substance use patients [40]. The groups were adapted to be an open, rolling format, and multiple meetings were held per week so that participants could complete all eight sessions during their 28-30 day stay. The study did not find significant post-treatment differences between conditions on primary outcomes (craving, mindfulness, and psychological flexibility).

Furthermore, the efficacy of MBRP with active and sham transcranial direct current stimulation (tDCS) was compared among individuals interested in decreasing their alcohol use [42]. Witkiewitz and colleagues' results demonstrated a decrease in drinks per drinking day over time among the sample as a whole and a significant dose effect for the number of groups attended. However, there were no effects of active versus sham tDCS on primary (drinks per drinking day) or secondary outcomes (percent heavy drinking days and alcohol cue reactivity). This suggests no additive effects of tDCS in enhancing MBRP among individuals seeking outpatient treatment for drinking. A secondary analysis of this trial was conducted to examine the impact of tDCS on hypersensitivity to alcohol cues (as measured by craving, negativity toward cues, and late positive potential) [31]. Brown and colleagues found that self-reported craving and late positive potential (LPP; an event-related brain potential that is thought to measure motivational and/or emotional salience) decreased when exposed to alcohol cues across all conditions over time. The magnitude of the reduction was associated with the number of MBRP sessions attended, such that participants attending a greater number of sessions reported decreased craving when exposed to alcohol cues.
Moderators of treatment outcome In an attempt to extend previous findings on MRBP efficacy, recent secondary data analyses of large-scale trials have aimed to identify subgroups who may benefit the most from MBRP. A secondary data analysis of two separate MBRP RCTs was conducted to examine potential moderators of efficacy [38]. In one sample [59], participants with high SUD severity and high levels of anxiety and depression that received MBRP reported 96\% fewer heavy drinking days and $94 \%$ fewer drug use days at 12 -month follow-up relative to relapse prevention (RP) therapy and TAU. Participants with high SUD severity and low levels of anxiety and depression that received MBRP reported 96\% fewer heavy drinking days at 12-month follow-up relative to relapse prevention and treatment as usual. In another sample [55], participants with high SUD severity and high anxiety/depression or high SUD severity and low anxiety/depression that received MBRP reported 99\% fewer alcohol and other drug use days than TAU participants. Roos and colleagues' results suggest that MBRP may be most effective for individuals with high SUD severity or for individuals with high SUD severity and high levels of co-occurring anxiety and depression symptoms.

Two recent analyses have also examined demographic factors as moderators of treatment outcome. Roos and colleagues conducted a secondary data analysis of an MBRP RCT [59] to examine individual gender and group gender composition as a moderator of efficacy [39]. Participants in the MBRP condition reported $84 \%$ fewer drug use days at 12-month follow-up relative to relapse prevention participants. There were no significant main effects of individual gender or group gender composition. There was a significant group by gender interaction such that individuals in MBRP groups with one-third or more women reported $100 \%$ fewer drug use days at 12-month follow-up relative to relapse prevention participants.

With respect to racial/ethnic identity, Greenfield and colleagues conducted a secondary analysis of the initial MBRP pilot trial [55] examining the role of individual identity and racial/ethnic group composition among individuals receiving an MBRP intervention after discharge from an intensive outpatient unit or inpatient treatment [37]. White participants in the MBRP condition had lower heavy drinking days relative to relapse prevention (control condition), but no treatment difference in drug use days. Minority participants did not have significant treatment differences in heavy drinking days but did have lower drug use days in the MBRP condition than control. Racial/ethnic group composition was a significant moderator with participants in groups that were more than half white exhibiting lower heavy drinking days in MBRP than control, whereas participants in 
groups that were more than half minority showed no treatment difference in heavy drinking days. The group composition interaction was non-significant for drug use days.

Mechanisms of behavior change Several recent studies have examined factors that mediate the effect of MBRP on substance use outcomes, including reductions in stress, impulsivity, craving, and stress reactivity. Davis and colleagues conducted a trial among lowsocioeconomic status young adults in a residential substance use treatment facility $(n=79)$ [33]. Participants were randomly assigned to receive either eight sessions of MBRP or eight social support group sessions (e.g., Alcoholics Anonymous) in addition to typical treatment during their residential stay. At post-treatment, young adults receiving MBRP reported lower levels of substance use (Cohen's $d=-0.58$ ), craving (Cohen's $d=-$ 0.58 ), and stress (Cohen's $d=-0.77$ ) relative to TAU participants. Stress reduction during treatment partially mediated observed outcome differences between MBRP and TAU for substance use, suggesting that the effects of MBRP on long-term substance use may be at least partially explained by reductions in stress.

The efficacy of MBRP at decreasing impulsivity was compared to TAU and 12-step/self-help among young adults in residential SUD treatment [34]. Davis and colleagues found that MBRP participants reported significantly lower negative urgency throughout treatment (Cohen's $d=-0.26)$ and maintained the improvement at follow-ups (Cohen's $d=-0.51$ and -0.21 ) relative to TAU. All participants reported significantly lower positive urgency during treatment and MBRP participants maintained these gains at follow-up (Cohen's $d$ range from -0.37 to -0.10 ). MBRP participants reported significantly lower lack of premeditation (Cohen's $d$ ranged from -0.66 to -0.43 ) and lack of perseverance (Cohen's $d$ ranged from -0.43 to -0.16 ) during treatment but did not maintain this change at follow-up. There were no differences between conditions on sensation seeking. Additionally, positive and negative urgency mediated the relationship between treatment and substance use, such that participants in the MBRP condition had greater reductions in urgency and were then less likely to use the substance over time. The results suggest that MBRP is an effective intervention for young adults with SUD in residential treatment and that positive and negative urgency may be a mechanism of change.

A secondary data analysis of two separate MBRP RCTs was conducted to examine if the finding of mindfulness mediating the effect of MBRP on craving replicates in a new sample of individuals who completed the same measures [36]. In one sample [55](Study 1), the effect of MBRP on psychological flexibility, craving, and mindfulness was small to medium (Cohen's d ranged from 0.08 to 0.48 ) and much smaller in the other sample ([59] Study 2; Cohen's d ranged from 0.03 to 0.21). In Study 1, participants had higher scores on these mindfulness measures at post-treatment relative to TAU, and the post-treatment latent mindfulness factor significantly mediated the associations between MBRP and craving. In Study 2, participants in the TAU condition had higher scores on the three mindfulness measures at posttreatment relative to MBRP, and there was no mediating effect of mindfulness.

Carroll \& Lustyk used a cognitive stressor to compare its impact on reactivity (measured by tonic and phasic heart rate variability, blood pressure, self-reported anxiety, self-reported craving, and heart rate) among adults who had completed 8 weeks of MBRP $(n=12)$, RP $(n=$ 12 ), or TAU (weekly process-oriented groups regularly provided by the community treatment agency; $n=10$ ) [32]. MBRP was related to higher levels of tonic and phasic heart rate variability, lower self-reported anxiety, and lower heart rate reactivity (than TAU only). There were no significant treatment effects on blood pressure or craving. Although formal mediation was not evaluated, the results suggest that participants who completed MBRP are still engaging with stress, but are cued to respond to stress in an adaptive, skillful manner. These processes might help to decrease substance use by increasing the use of adaptive coping skills.

\section{Mindfulness oriented recovery enhancement (MORE) Overview of protocol}

Mindfulness Oriented Recovery Enhancement (MORE) integrates elements of mindfulness training, cognitive restructuring, and positive psychology to address the factors theorized to maintain SUD [22]. More specifically, the three foundational elements of MORE are mindfulness, reappraisal, and savoring [22]. Mindfulness is described as a way to identify triggers and break the habit of automatic engagement in substance use in response to those triggers. Reappraisal is the process by which an individual reassesses a stressful event to be something more positive and constructive, as opposed to negative and destructive. Savoring is the act of focusing one's attention on the positive, pleasing, and growth-inducing elements of an event. These foundational elements of MORE are the guiding principles that influence the tensession protocol.

The standard protocol includes 10, 120-min group sessions that guide participants through the three foundational elements. The first three sessions of the MORE protocol are focused on laying the groundwork for the course by introducing the three foundational elements of MORE. In addition to introducing the practice of mindfulness, the first session presents the idea of 
automaticity, or "autopilot," and the relationship between automaticity and engaging in substance use [22]. During sessions one through three, clients are asked to engage in a variety of experiential practices that range from focused attention of the breath, reframing an ordinary encounter on the street, to mindfully savoring an object in one's immediate field of vision.

Sessions four through seven are centered around the Buddhist notion of impermanence, and the themes build upon the foundation of mindfulness. Session four and five are concentrated on the role that craving plays in substance use. Clients are asked to see the fleeting nature of any feeling, craving included, and to allow those temporary feelings to move through one's body and mind without engaging with them or trying to change them. Clients experience the nature of craving when interacting with an object that is perceived as a treat (a piece of chocolate), then observe those same feelings of craving during a stressful situation [22]. Session six introduces the role that aversion plays in the recovery process, and how urges can be intensified when one tries to push away thoughts about drugs or alcohol. Session seven builds upon the nature of impermanence by highlighting the fleeting nature of our physical bodies and stresses the deleterious effects that toxic substances and health-risk behaviors have on our fragile existence. The primary exercise used during this session is the Impermanent Body Exercise. This practice asks the participants to bring an awareness to the facts that we will all get sick, we will all age, and we will all eventually die.

The final three sessions focus on how to interact with the environment following treatment and navigate back to the three core components of MORE. Session eight revisits the concept of autopilot and focuses on how one could react differently to a situation that would have previously driven engagement in substance use. The awareness of triggers and the use of mindfulness to break the chain of automatic action is the key focus of this session. Session nine returns to savoring, and highlights the beautiful nature of the world around us and how all things are connected in some way. Clients are encouraged to see the interdependent nature of life and simply to enjoy that beautiful connection we all share. The primary practice used to demonstrate this interconnection is the Tasting Interdependence exercise. In this practice, clients are given a raisin and are asked to follow the span of that raisin's life from a grape seed planted into the soil up until the current moment as a dried piece of fruit. Session ten closes the course by recapping what has been learned, and how that new knowledge can be integrated into daily life.

MORE was originally developed and tested during an NIH-funded Stage 1 RCT for alcohol dependence. That study demonstrated that MORE could significantly improve physiological recovery from stress and drinking-related triggers [22]. Since that initial pilot trial of the MORE protocol, the program has been adapted and modified to fit the needs of various addictive behaviors such as opioid use disorder (OUD), internet gaming disorder, and chronic pain with prescription misuse.

\section{Literature review}

One of the primary processes that MORE targets is the urge to engage in substance use (i.e., craving). A largescale RCT compared MORE to Cognitive Behavioral Therapy (CBT) and TAU in 180 male patients with cooccurring psychiatric and substance use disorders [15, 46]. Garland and colleagues found reductions in craving in all three conditions, with greater reductions in MORE than CBT, but not TAU. In addition to a reduction in craving, MORE was associated with greater decreases in post-traumatic stress symptoms versus both CBT and TAU.

Several recent trials have leveraged ecological momentary assessment (EMA) to explore the impact of MORE on craving, pain, and affective states. In one stage 1 RCT, EMA was utilized to examine the effects of MORE versus TAU on opioid craving, pain, and positive affect on individuals enrolled in methadone maintenance therapy (MMT) [51]. Compared to TAU, participants in MORE reported significantly greater decreases in opioid wanting (44\% decrease) and opioid urge (50\% decrease). EMA data also showed that participants in MORE reported a greater number of cravings $(n=303)$ than participants in TAU $(n=87)$, but MORE participants reported those cravings as being significantly less intense than those experienced by individuals in TAU. Participants in MORE also reported having 129\% greater selfcontrol over cravings than participants in TAU. Garland and colleagues also collected EMA data from a subset of individuals $(n=55)$ from a larger RCT to test the efficacy of MORE versus a support group (SG) at reducing perceived pain and improving affective state [48]. Participants were chronic pain patients with daily, to near daily, use of prescription opioids. Through the 8-weeks of the intervention, participants in the MORE group showed a $7 \%$ reduction in pain, compared to a $3 \%$ increase among the SG; thus pain intensity improved over the 8 weeks of MORE relative to the SG. Over the same duration, participants in MORE were 2.75 times more likely to be emotionally regulated than participants in the SG. Mean opioid misuse scores decreased during treatment by 7.06 in the MORE group and by 3.25 in SG.

Another mechanism that the MORE program may affect is cue reactivity. One study evaluated the impact of MORE versus a SG on the late positive potential (LPP) index of opioid cue-reactivity relative to reactivity 
to neutral cues in 135 individuals with chronic opioid use [52]. The LPP was used to measure prolonged attention to the opioid cues. Garland and colleagues found that for individuals in the SG, post-treatment LPP remained significantly higher in response to opioid cues compared to the neutral cues. Participants in MORE exhibited significantly greater decreases in the LPP response to opioid cues and also exhibited greater increases in the LPP response to natural reward cues from pre- to post-treatment. In another study, Garland and colleagues tested the relative responsiveness to natural reward and opioid cues in 51 individuals who used prescription opioids [49]. There was a significant group $\times$ time effect on heart rate variability (HRV) responsivity, indicating that compared to the SG, the MORE group experienced significantly greater increases in HRV responsivity in response to drug elicited cues.

Garland and colleagues also conducted a secondary data analysis to compare the effects of MORE on opioid attentional bias (AB) compared with a SG in 115 individuals who used prescription opioids [47]. The pre-treatment opioid $A B$ significantly moderated the effect of the treatment condition on posttreatment opioid $A B$, indicating that the effects of treatment significantly differed by pre-treatment opioid $\mathrm{AB}$. The post-treatment opioid $\mathrm{AB}$ score for MORE and SG participants (with pre-treatment opioid $A B$ as a covariate) revealed a statistically significant effect of the treatment condition, with MORE participants showing significantly lower levels of opioid $A B$ at post-treatment than SG participants. The reductions in opioid $\mathrm{AB}$ over the course of treatment predicted lower levels of opioid misuse at 3-month follow-up. All of these findings suggest that MORE may sever associations between conditioned drug cues and their reward value via bottom-up mechanisms and that decreasing attentional fixation on opioid cues may reduce the risk of future opioid misuse.

In an analysis of proximal outcome data collected from a larger RCT, Garland and colleagues looked at the effectiveness of MORE versus a SG on pain severity, opioid misuse, positive affect, meaning in life, and selftranscendence in 95 opioid treated chronic pain patients [50]. They found that there was a significant effect of intervention group on change in positive affect, meaning in life, savoring, and self-transcendence, indicating that MORE increased these attributes more so than the SG. For the clinical variables, there was a significant effect of intervention group on change in pain at post-treatment and change in opioid misuse risk by 3-month follow-up, such that MORE resulted in greater improvements in these variables than did the SG.

\section{Mindful awareness in body-oriented therapy (MABT) Overview of protocol}

Mindful Awareness in Body-Oriented Therapy (MABT) is a manualized, mindfulness-based approach that is designed to teach interoceptive skills for self-care [23]. Interoception is the ability to process signals that originate in the body and is broadly described as the overall sensations, or state, of the body [60]. MABT is different from most mindfulness-based practices in that it specifically focuses on teaching the client mind-body skills that are designed to help with a dysregulated awareness of sensory information. There appears to be a link between a deficient interpretation of sensory information and poor emotion regulation [24]. The intervention involves eight, 90-min group sessions over eight consecutive weeks. To meet the primary aim of MABT, the course is broken down into three specific stages. Stage 1 (sessions 1 and 2) is geared toward identifying bodily sensations (identification). Often clients are unaware that physical sensations can be identified, and so, the first two sessions are aimed at teaching body literacy through a response to expected sensations (i.e. the client grasping her arm and feeling the pressure created) [24].

Stage 2 of the MABT program (sessions 3 and 4) is directed at learning and developing strategies for interoceptive awareness (access), which is a more advanced aspect of body literacy that adds upon the skills learned in the first stage [23]. This stage focuses on sensations that are still quite overt and apparent, such as the sensation of breathing in and out, but clients are beginning to refine their bodily awareness. Clients progress from sensing continuous movements in the body to perceiving specific areas of the body (chest, foot, etc.) to develop the skills of interoception.

The final stage of MABT (sessions 5 through 8) is aimed at developing the ability to maintain/sustain interoceptive awareness throughout one's daily life (appraisal) [23]. The ability to sustain this awareness of bodily sensations allows the client to detach oneself from the uncomfortable physical sensations that may be occurring in a situation, and allow themselves to reframe the experience more positively. The ability to investigate and determine the origin of the physical sensations that are occurring during a particular event, or emotional state of being, allow the client to take charge of their experience and perform the necessary actions for self-care that are more productive than engaging in substance use.

\section{Literature review}

In a large-scale RCT of 217 women enrolled in three separate outpatient treatment facilities, researchers determined if MABT resulted in improved interoceptive awareness and mindfulness skills (primary outcomes), 
emotion regulation (self-report and psychophysiology), symptomatic distress (e.g., depression and traumarelated symptoms), and substance use and craving compared with women's health education (WHE), and TAU alone [23]. Price and colleagues found that MABT produced significantly greater improvements in mindfulness from pre- to post-intervention among participants who completed six or more sessions, as compared to the other groups. However, this effect was not significant in ITT analyses, including all participants with any available data. MABT also produced greater improvements in most factors of interoceptive awareness, including noticing, attention regulation, emotional awareness, selfregulation, body listening, and trust, as compared to the other two groups. However, MABT did not result in greater improvements in depression or trauma symptoms, both secondary outcomes, compared to the other two treatment groups (symptoms improved in all groups). Participants in both MABT and WHE conditions, compared with TAU, showed significantly greater improvement in the proportion of days abstinent; however, there were no significant differences across groups in relapse or craving.

A longitudinal follow-up of the previously mentioned RCT examined the number of days abstinent, overall psychological well-being, interoceptive awareness, and mindfulness skills across the three conditions through 12-month follow-up assessments among 187 of the original 217 participants [53]. Looking at the primary outcome of days abstinent from substance use among those who completed at least six sessions of treatment, the MABT and WHE groups showed a greater number of days abstinent compared to the TAU group at the 6month and 12-month follow-up. The improvements in the number of days abstinent among MABT participants were maintained from 3 to 12 months, whereas TAU and WHE showed continuous declines in abstinent days, particularly at 12 months. There were no group differences observed for MABT vs. WHE in the percent of days abstinent or relapse at any assessment time-point.

\section{Mindfulness-based addiction treatment (MBAT) Overview of protocol}

Mindfulness-Based Addiction Treatment (MBAT) is a protocol that closely follows the procedures and rationale of Mindfulness-Based Cognitive Therapy (MBCT). Wetter and colleagues took most of the material from the MBCT handbook, but they removed the depression focused content and replaced it with information regarding substance use (e.g. smoking cessation material, alcohol abstinence, etc.) [25]. Three primary aims lead the eight, 120-min group sessions, including: (1) becoming aware of thoughts, emotions, and physical sensations from moment to moment, (2) creating a different way of relating to those thoughts, emotions, and physical sensations, and (3) using ones new-found awareness to separate oneself from those various occurrences that are arising [25]. Like the other mindfulness-based protocols reviewed, the primary aim of MBAT is to awaken clients to the automatic nature of substance use by teaching them the skills of noticing present moment feelings and sensations and detaching themselves from urges to engage in substance use.

The MBAT course is broken down into two primary sections. Sessions $1-4$ are geared toward teaching clients how to direct and focus their attention [26]. This is done using a crawl-walk-run framework that has participants learn to focus their attention on something that is constantly occurring regardless of whether attention is paid to it or not, such as the sensation of breathing. Sessions 5-8 are aimed at using the skills of focused attention to help navigate through difficult situations that are related to substance use. Clients are taught specific techniques, primarily one titled "breathing space," to find some room between stimulus and response [25]. For example, the group may be lead through a visualization exercise in which clients picture a situation where engaging in substance use would have been automatic. Clients are asked to pause the scenario just before visualizing engagement in substance use and are directed to instead observe the breath. This pause allows clients to detach from their feelings of craving and gives them the necessary space to investigate what is arising in them physically and emotionally.

\section{Literature review}

In a large-scale RCT, Vidrine and her research team wanted to test the effects of the MBAT protocol on smoking cessation compared with CBT and a usual care (UC) condition comprised of brief individual counseling sessions based on the Treating Tobacco Use and Dependence Clinical Practice Guideline [25] among 412 adult smokers. Results from the study found that participants in the MBAT condition had a higher percentage of 7-day point percentage abstinence compared with the other two groups, but the difference was not statistically significant $(32.5 \%$ in UC, $39.1 \%$ in CBT, and $42.1 \%$ in MBAT at 4 weeks post quit day). Among participants classified as still smoking at final treatment sessions, recovery of abstinence was examined. The MBAT condition had significantly higher recovery rates after relapse than both the CBT and UC groups 1 week following the end of treatment (13.2\% in UC, $7.0 \%$ in CBT, and $26.8 \%$ in MBAT) and 26 weeks post-quit day ( $0 \%$ in UC, $3.5 \%$ in CBT, and $7.1 \%$ in MBAT).

Additional data collected during the parent study described above examined the cognitive and affective mechanisms underlying MBAT versus $\mathrm{CBT}$ and $\mathrm{UC}$ for 
smoking cessation, including: positive and negative affect, dependence, withdrawal, craving, agency, and subjective bias towards cigarettes [26]. Spears and colleagues found that members in the MBAT group perceived greater volitional control over smoking and lower volatility of anger than CBT and UC. Although the mediators of the effect of MBAT versus $\mathrm{CBT}$ on lapse recovery were not observable because of small subsamples, greater perceived volitional control over smoking and lower affective volatility could be mechanisms explaining why MBAT may be a better option than both CBT and UC for promoting lapse recovery in smokers. There were no other significant differences between MBAT and CBT, nor were there significant indirect effects of MBAT versus CBT, suggesting that mindfulness and cognitive-behavioral approaches may similarly influence several of the psychosocial mechanisms implicated in tobacco dependence.

\section{Mindfulness training for smoking cessation (MTS) Overview of protocol}

Mindfulness Training for Smoking Cessation (MTS) is built upon the foundational ideas outlined in both MBSR and MBRP, but was tailored specifically for individuals who are trying to stop smoking [27]. The eight-week protocol is broken down into eight, 90-min group sessions. The primary aims of the MTS course are to (1) teach participants the skills of observing thoughts, feelings, and sensations as they are occurring in the present moment, and (2) hone the ability to accept and allow the current situation to be just as it is [16].

Sessions one and two introduce participants to how smoking has become a learned behavior through habituation and associative learning [27]. The first session introduces clients to the visceral feelings of craving, and how the urge to smoke manifests itself in the body. Session two presents the primary tool that participants can use during times of craving. The acronym RAIN (Recognize, Accept, Investigate, and Note) is used as an easy-to-remember mnemonic that clients can use when feelings of craving arise [27]. Session three introduces a traditional meditation practice, loving-kindness (metta meditation), where clients direct well wishes towards oneself and others. This practice is introduced to help clients engage with stress and negative feelings with an empathetic mind, and a kind acceptance of those feelings. Session four is the most unique aspect of the MTS course, and this is the session that sets it apart from many other MBIs, in that it encourages a formal declaration of abstinence. This session is aptly named "quit day," and clients are asked to commit to not smoking, and are given techniques to build upon the skills of noticing the sensations that are arising in the body, primarily craving, during initial abstinence from smoking [16].
Sessions 5-7 introduce participants to how specific triggers can hinder long-term abstinence and how mindfulness practices (mindfulness of breath, RAIN, and mindful walking) can be integrated into one's daily life to help them identify triggers and avoid smoking. Session eight summarizes all of the tools and techniques learned throughout the program and explores ways of maintaining mindful awareness in the future.

The feasibility and effectiveness of MTS was initially tested in 2013 with 55 young adult smokers [16]. This small scale pilot study found that point prevalent smoking abstinence was higher in MTS than controls, though the difference was not significant. The promising, yet underpowered results of this study have encouraged larger-scale research on the effects of the MTS protocol.

\section{Literature review}

An RCT conducted by Kober and colleagues compared the effects of MTS on smoking cessation with the American Lung Association's Freedom from Smoking (FFS) program [16]. More specifically, they looked at whether stress reactivity, including neural activity during stressful scripts, related to smoking cessation directly after treatment as well as at the 3-month post-treatment follow-up. The sample included 23 smokers between the ages of 18-60 who smoked more than 10 cigarettes per day. Both treatments reduced smoking, but the MTS group demonstrated a greater rate of reduction in cigarette use during treatment, which was maintained during the 3-month post-treatment follow-up. The MTS group showed a trend toward greater 1-week point prevalence abstinence at the end of treatment (55\% vs. $23 \%$ ), and this difference became statistically significant at the 17 -week follow-up ( $44 \%$ vs. $7 \%$ ). Across all participants, neural activity during the stressful scenarios in the bilateral amygdala, anterior insula, mid insula, hippocampus, parahippocampal gyrus, thalamus, middle occipital gyrus, midbrain, cerebellum, and right posterior insula, as well as a second region spanning the midline across cuneus/precuneus and posterior cingulate cortex, were correlated with less reduction in cigarettes smoked per day at post-treatment [16]. During stressful scenarios, participants in the FFS group (vs. the MTS group) exhibited increased neural reactivity in several brain regions including the left amygdala, anterior, middle, and posterior insula, and bilateral portions of parahippocampal gyrus and hippocampus, putamen, thalamus, midbrain and cerebellum [16]. The MTS group did not show greater neural reactivity in any region during the stressful scenarios. 


\section{Moment-by-moment in Women's recovery (MMWR) Overview of protocol}

Moment-by-Moment in Women's Recovery (MMWR) was developed as an adaptation of MBSR, specifically designed for low income, racially, and ethnically diverse women currently enrolled in residential SUD treatment [28]. MMWR is intended to be administered in a residential setting, a feature that distinguishes it from other MBIs [28].

MMWR is comprised of eight, 90-min group sessions, and one four-hour intensive retreat day. The first session is an introduction to mindfulness, including the definition of mindfulness and how mindfulness can play an integral role in the recovery process [29]. Sessions two and three are focused on demonstrating the automatic and constant nature of thoughts, feelings, and emotions that arise throughout the day. The theme for session three is geared at empowering clients, and showing them that the perception of their environment can dictate how a situation appears (i.e. positive or negative).

Sessions 4-6 are the core lessons dedicated to learning and practicing mindfulness skills. Session four is devoted to understanding the role that negative emotions can play in relapse [29]. The emotions of anxiety, fear, and panic are the focus of this session, and clients are asked to engage in experiential exercises to practice the skill of mindfulness during these uncomfortable situations. Session five is focused on the emotions of guilt and shame [29], which often play a role in relapse. Clients are guided through a visualization exercise to practice using mindfulness while experiencing these emotions. Practicing these skills while in a safe environment helps to give clients confidence in their new skills, and helps to bolster their confidence in their ability to prevent relapse. Session six is dedicated to using mindfulness while interacting with others, including mindful communication and the importance of positive self-talk [29].

Session seven is a four-hour "retreat" day that allows clients to immerse themselves in silent meditation to practice the skills they have been learning throughout the course. Session eight is aimed at teaching participants how to engage mindfully with anger and violence. The emotion of anger is the focus of this session, and participants are asked to identify and observe their own triggers for anger. In the final session, all of the activities and skills that were presented during the course are reviewed to solidify and sustain the concepts learned.

\section{Literature review}

The MMWR intervention has a very specific and niche target population that lends itself well to researching the effects of the intervention without many confounding variables. In a large-scale RCT, Black and colleagues tested the efficacy of MMWR on improving SUD treatment retention when applied as an adjunctive intervention to residential treatment compared with a neurobiology of addiction psychoeducation course (control condition) [17]. The sample was comprised of 200 adult women diagnosed with SUD and admitted to the residential SUD treatment program study site. The primary outcomes of the study were discharge status (if a client developed the necessary skills to complete residential treatment), days until discharge (the number of days a client remains in residential treatment beginning with the first day of the intervention and ending at day 150), and self-report measures of mindfulness disposition, perceived stress, distress tolerance, emotion regulation, psychological distress, affect, and drug and alcohol craving [17]. The count of women defined as treatment completers (client completed SUD treatment) was 43/100 in the MMWR group versus 31/100 in the control group. The count of women in-residence (client still at the residential treatment site at day 150) was $15 / 100$ in the MMWR group and 27/100 in the control group. Among the 84 treatment non-completers (client left the facility before the end of treatment), satisfactory progress was made by $16 / 42$ women in the MMWR group and 10/42 women in the control group. However, there were no differences between conditions for any of the self-report measures, including craving.

\section{Conclusions}

The purpose of this manuscript was to provide a session-by-session overview of treatment protocols of MBIs and to systematically review randomized controlled trials (RCTs) of MBIs for SUD, with a focus on studies published after 2017 to update a previous systematic review conducted by Li et al. [19]. Key findings on clinical implications, current limitations in the field, and suggestions for the road ahead are presented below.

There is a variety of MBIs in the field of SUD treatment. All of the protocols we reviewed have some commonalities that target factors maintaining SUD. Bringing one's attention to the immediate experience of the present moment is the foundation upon which all of these interventions are based. Regardless of the techniques employed, the goal of mindfulness is to stop (momentarily) rumination about past events and the planning of future actions. Most of the interventions we reviewed have both formal and informal meditation practices that are designed to encourage participants to break the cycle of thoughts and stop the process of acting on autopilot. One of the key components of SUD is a desire (i.e. craving) to engage in substance use due to habit or a desire to increase momentary enjoyment or avoid discomfort. One of the primary aims of the MBIs we reviewed is bringing focused attention to whatever is arising in the present moment (i.e. craving, sadness, 
pain, etc.) without judgment, and without a need to engage with, including actively avoiding, those feelings.

Despite sharing some commonalities, many of these programs have been designed for different populations and substances. One of the cornerstones of MBRP is becoming aware of triggers and finding ways to cope with cravings. This focus on triggers and craving may suggest that MBRP is most beneficial for an individual in the earlier stages of changing their substance use. MTS, MBAT, and MMWR all have strong foundational links to MBSR. These three protocols are primarily centered around bringing attention to the constant stream of thoughts everyone has during the day. This observation of thoughts is designed to cut through the automaticity of SUD. MABT is an intervention centered around interoception and becoming aware of the deleterious effects of engaging in addictive behavior. This intervention can be beneficial at teaching participants self-care practices to take the place of substance use. The different focal points that these protocols have may suggest that they are better suited for later stages of substance use behavior change. Future research should focus on these differing focal points to determine the best time in the recovery process to deliver these differing MBIs.

The growing field of MBIs for SUD has produced mixed results. While some studies have yielded positive outcomes, others have found that MBIs perform no better than existing treatments or control conditions. Some of the findings from recent MBRP research show that this protocol may be quite effective for individuals with high SUD severity or high levels of co-occurring anxiety and depression symptoms (e.g., [31, 35]). Several reviewed studies also found promising effects of MBIs on a range of psychiatric outcomes, including depression and anxiety symptoms (e.g., [26, 35, 44]), psychological flexibility (e.g., [40]), posttraumatic stress disorder symptoms, and positive affect (e.g., [15]). With the high prevalence of SUD comorbid with other mental health conditions, positive results in populations with high levels of depression and anxiety symptoms, as well as decreases in psychiatric distress over MBIs, are encouraging. However, not all studies examining secondary psychiatric distress outcomes evidenced benefits of MBIs in comparison to control groups (e.g., [41]), and therefore additional research is needed to determine particular components of MBIs and/or the dose of mindfulness practice that best targets psychiatric distress.

Another positive outcome we found was the beneficial effects that MBIs have on cue-reactivity and attentional bias [47, 48, 52]. Mitigating the sustained, motivated focus on substance-related cues, as well as an individual's reaction to these cues, may prove to be one of the most beneficial aspects of MBIs in the treatment of SUD. An additional factor that MBIs appear to target is perceived stress. Many of the protocols we reviewed demonstrated that participants had more volitional control of stressful situations that may have previously led them to engage in substance use [23, 32, 33]. These findings could be linked to the process of non-judgmental awareness that all of the protocols encourage.

Despite some of the more positive findings, some of the articles we reviewed showed no significant differences between MBIs and other evidenced-based treatments. Many studies found little to no effect on secondary outcomes such as positive and negative affect, emotion regulation, or mindfulness scores [23, 36, 41]. This lack of improvement in putative mechanisms of change may be due to the fact these are not the right mechanisms to be investigating. With the large number of positive outcomes that many of these studies did find, it is hard to imagine that these MBIs are not positively impacting behavior change on some level. One challenge in interpreting outcomes from MBIs is the varying definitions of mindfulness that have been utilized, both as an outcome and as content in MBIs. Therefore, identifying the most appropriate outcomes and mediators to examine is difficult when there is such a broad spectrum of definitions for the behavioral mechanisms targeted by MBIs. Future research must focus on what underlying behaviors mindfulness targets to develop and use appropriate evaluation tools and better fidelity measures to determine the effects of these interventions. Hopefully, future research may be able to shed some light on the best mechanisms of change to be investigated.

However, we found several limitations that make it difficult to draw firm conclusions. First, there is a pressing need for large scale RCTs with equivalent control conditions and long-term follow-ups. Many of the existing studies have small sample sizes with limited generalizability, lack of equivalent time/effort controls, and the results of the studies have small effect sizes with large confidence intervals. Another factor that needs to be controlled for is treatment fidelity. With the ability to administer certain MBIs after having simply bought the treatment manual or taken a weekend course, there is a need for uniformity when administering these interventions. Finally, one of the biggest questions yet to be answered in the study of MBIs is the minimum effective dose required to exhibit the desired behavior change. Many of the current MBIs follow the structure of the original MBSR protocol developed by Kabat-Zinn in the early 1980s with little variation. The entire field of mindfulness research would benefit from testing variations from the standard 8-week intervention initially developed to determine the appropriate dose-response of meditation in these clinical populations. We believe that meditation is a dose-dependent intervention, but future research must determine the minimum threshold (i.e. 
time dedicated to meditation in both formal and informal practice) to see the expected improvements in psychiatric, cognitive, and substance use outcomes. Conducting large RCTs with stringent experimental controls may help provide a clearer picture of the effectiveness of MBIs.

Results of the present review are also qualified by several limitations. We limited ourselves to the search terms utilized in a previous review by $\mathrm{Li}$ and colleagues [19], and therefore may have overlooked relevant articles that were not captured by these search terms. Similarly, we chose to focus on SUD, specifically, as opposed to addictive behaviors, broadly. Examining MBIs for behavioral addictions is an emerging area of research and future reviews of these studies may provide further insight into the mechanisms of MBIs for addictive behaviors. Lastly, the results of the present review were purely based on a qualitative, not quantitative, analysis. Although we chose to provide a narrative review of the efficacy of MBIs for SUD, best practices for these interventions, and future research directions (including future meta-analyses) in this area will continue to provide critical information for understanding the effectiveness of MBIs for addictive behaviors.

Mindfulness has been used for centuries to help with the fleeting and sometimes difficult nature of the human mind, and these practices have recently proliferated in Western medicine, particularly to mitigate mental health disorders, including SUD. A majority of the MBIs for SUD reviewed use similar foundational concepts to help clients bring focused attention to their daily lives, indicating that the aspects of mindfulness are likely viable interventions for SUD. Continued research, including large-scale RCTs with equivalent control groups and long-term follow-ups, will clarify the function mindfulness can play in the treatment of SUD, and will also help develop the most effective way to integrate mindfulness into existing SUD treatment programs. Widespread interest in mindfulness and its benefits in the clinical setting continue to proliferate, and results from future research will show us the best contexts and settings for its use. Further, given evidence that MBIs are at least as effective, and in some cases more effective, than existing treatments would suggest that these interventions are ready for widespread dissemination. Future research should focus on approaches to increase the dissemination and implementation of MBIs into more community treatment settings [61]. Cost-effectiveness analyses of MBIs for SUD would also be an important next step to inform policy makers and tax payers. It is potentially the case that investing in more SUD treatment programs that offer MBIs will potentially yield cost savings, as has been shown for other MBIs [62].

\section{Abbreviations}

AAQ: Acceptance and Action Questionnaire; AAQ-SA: Acceptance and Action Questionnaire - Substance Abuse; AB: Attentional Bias; ACT: Acceptance and Commitment Therapy; AIPQ: Affective Information Processing Questionnaire; ASI: Addiction Severity Index; BAI: Beck Anxiety Inventory; BDI: Beck Depression Inventory; BIS: Barratt Impulsiveness Scale; BPI: Brief Pain Inventory; CBT: Cognitive Behavioral Therapy; CES-D: Center for Epidemiologic Studies Depression Scale; CM: Contingency Management; COMM: Current Opioid Misuse Measure; DASS: Depression Anxiety Stress Scales; DBT: Dialectical Behavior Therapy; DERS: Difficulties in Emotion Regulation Scale; DrInC: Drinker Inventory of Consequences; DUD: Drug Use Days; EEG: Electrocardiography; EMA: Ecological Momentary Assessment; FFMQ: Five Facet Mindfulness Questionnaire; FFMQ-BR: Five Facet Mindfulness Questionnaire-Brazil; FFS: Freedom from Smoking; FIM: Functional Independence Measure; fMRI: Functional Magnetic Resonance Imaging; GAIN: Global Appraisal of Individual Needs; HAD: Hospital Anxiety and Depression; HCQ: Heroin Craving Questionnaire; HDD: Heavy Drinking Days; HE: Health Education; HR: Heart Rate; HRV: Heart Rate Variability; HSI: Heaviness of Smoking Index; IRR: Incidence Rate Ratio; ITT: Intent To Treat; LPP: Late Positive Potential; MAAS: Mindful Attention Awareness Scale; MAIA: Multi-dimensional Assessment of Interoceptive Awareness; MABT: Mindful Awareness in Body-Oriented Therapy; MBAT: Mindfulness-Based Addiction Treatment; MBCT: Mindfulness-Based Cognitive Therapy; MBI: Mindfulness-Based Intervention; MBRP: MindfulnessBased Relapse Prevention; MBSR: Mindfulness-Based Stress Reduction; MLQ: Meaning in Life Questionnaire; MMT: Methadone Maintenance Therapy; MMWR: Moment-by-Moment in Women's Recovery; MORE: Mindfulness Oriented Recovery Enhancement; MTS: Mindfulness Training for Smokers; NADA: Non-dual Awareness Dimensional Assessment; OUD: Opioid Use Disorder; PACS: Penn Alcohol/Drug Craving Scale; PANAS: Positive and Negative Affect Schedule; PASS: Pain Anxiety Symptoms Scale; PCL: PTSD Checklist; PSS: Perceived Stress Scale; PSS-SR: PTSD Symptom Scale - SelfReport; PTSD: Post-traumatic Stress Disorder; QSU: Questionnaire of Smoking Urges; RAIN: Recognize, Accept, Investigate, and Note; RCT: Randomized Controlled Trial; RSA: Respiratory Sinus Arrhythmia; SBQ: Subjective Attentional Bias Questionnaire; SDS: Severity and Dependence Scale; SFS: Substance Frequency Scale; SES: Smoking Self-Efficacy Scale; SG: Support Group; ST: Standard Treatment; SUD: Substance Use Disorder;

TAU: Treatment as Usual; tDCS: Transcranial Direct Current Stimulation; TLFB: Timeline Followback; UC: Usual Care; UPPS-P: Urgency, Premeditation, Perseverance, Sensation Seeking, and Positive Urgency; WBSI: The White Bear Suppression Inventory; WHE: Women's Health Education; WISDM: Wisconsin Inventory of Smoking Dependence Motives; WSWS: Wisconsin Smoking Withdrawal Scale

\section{Acknowledgments}

Not applicable.

\section{Authors' contributions}

JRK completed the initial literature search. JRK, FJS, and VRV reviewed all of the manuscripts for applicability and inclusion for the systematic review and contributed to the first draft of the manuscript. KW developed the study concept and provided critical revisions. The authors approved the final version of the paper for submission.

\section{Funding}

This work was supported by the National Institutes of Health, award numbers R01AA025539 and T32AA018108. The content is the sole responsibility of the authors and does not necessarily represent the official views of the National Institutes of Health.

\section{Availability of data and materials \\ Not applicable.}

Ethics approval and consent to participate Not applicable. 


\section{Competing interests}

The authors declare that they have no competing interests.

Received: 21 May 2020 Accepted: 16 July 2020

Published online: 29 July 2020

\section{References}

1. Substance Abuse and Mental Health Services Administration. Key substance use and mental health indicators in the United States: results from the 2017 National Survey on drug use and health. Rockville: Department of Health and Human Services; 2018.

2. NIDA. Trends \& statistics; 2020.

3. Volkow ND. Principles of drug addiction treatment. Heal (San Fr)The Institute. 2009;12:1-80.

4. Kabat-Zinn J. Wherever you go, there you are : mindfulness meditation in everyday life. 1st ed Hyperion; 1994

5. Hanh N, Hanh TN. The heart of the Buddha's teaching: transforming suffering into peace, joy, \& liberation : the four Noble truths, the Noble eightfold path, \& other basic Buddhist teachings. Berkeley: Parallax Press; 1998.

6. Kabat-Zinn J, Lipworth L, Burney R. The clinical use of mindfulness meditation for the self-regulation of chronic pain. J Behav Med. 1985;8:16390.

7. Kabat-Zinn J, Lipworth L, Burney R, Sellers W, Brew M. Reproducibility and four year follow-up of a training program in mindfulness meditation for the self-regulation of chronic pain. Pain. 1984;18:S303.

8. Kabat-Zinn J. An outpatient program in behavioral medicine for chronic pain patients based on the practice of mindfulness meditation: theoretical considerations and preliminary results. Gen Hosp Psychiatry. 1982;4:33-47.

9. Segal ZV, Williams JMG, Teasdale JD. Mindfulness-based cognitive therapy for depression : a new approach to preventing relapse. New York: The Guilford Press; 2002.

10. Cullen M. Mindfulness-based interventions: an emerging phenomenon. Mindfulness (N Y). 2011;2:186-93.

11. Levy KN, McMain S, Bateman A, Clouthier T. Treatment of borderline personality disorder. Psychiatr Clin North AmGuilford Publications. 2018;41: $711-28$.

12. Hayes SC, Strosahl KD, Wilson KG. Acceptance and commitment therapy: an experiential approach to behavior change. New York: Association for Advancement of Behavior Therapy; 1999.

13. Bowen S, Chawla N, Witkiewitz K. Mindfulness-based relapse prevention for addictive behaviors. In: Baer RA, editor. Mindfulness-based treat approaches Clin Guid to Evid base Appl. 2nd ed. San Diego: Elsevier Academic Press; 2014. p. 141-57.

14. Witkiewitz K, Marlatt GA, Walker D. Mindfulness-based relapse prevention for alcohol and substance use disorders. J Cogn PsychotherSpringer Publishing. 2005:19:211-28.

15. Garland EL, Roberts-Lewis A, Tronnier CD, Graves R, Kelley K. Mindfulnessoriented recovery enhancement versus CBT for cooccurring substance dependence, traumatic stress, and psychiatric disorders: proximal outcomes from a pragmatic randomized trial. Behav Res TherElsevier Science. 2016;77: 7-16.

16. Kober $\mathrm{H}$, Brewer JA, Height $\mathrm{KL}$, Sinha R. Neural stress reactivity relates to smoking outcomes and differentiates between mindfulness and cognitivebehavioral treatments. NeuroimageElsevier. 2017;151:4-13.

17. Black DS, Amaro H. Moment-by-moment in Women's recovery (MMWR): mindfulness-based intervention effects on residential substance use disorder treatment retention in a randomized controlled trial. Behav Res TherElsevier Science. 2019;120:120.

18. Tiffany ST, Drobes DJ. Imagery and smoking urges: the manipulation of affective content. Addict BehavElsevier. 1990;15:531-9.

19. Li W, Howard MO, Garland EL, McGovern P, Lazar M. Mindfulness treatment for substance misuse: a systematic review and meta-analysis. J Subst Abus Treat. 2017;75:62-96

20. Goldberg SB, Tucker RP, Greene PA, Davidson RJ, Wampold BE, Kearney DJ, et al. Mindfulness-based interventions for psychiatric disorders: a systematic review and meta-analysis. Clin Psychol Rev. 2018;59:52-60.

21. Moher D, Shamseer L, Clarke M, Ghersi D, Liberati A, Petticrew M, et al. Preferred reporting items for systematic review and meta-analysis protocols (PRISMA-P) 2015 statement. Syst Rev. 2015;4:1.
22. Garland EL, Baker AK, Riquino MR, Priddy SE. Mindfulness-oriented recovery enhancement. In: Handb mindfulness-based program mindfulness Interv from Educ to heal Ther. Abingdon: Routledge; 2019.

23. Price CJ, Thompson EA, Crowell SE, Pike K, Cheng SC, Parent S, et al. Immediate effects of interoceptive awareness training through mindful awareness in body-oriented therapy (MABT) for women in substance use disorder treatment. Subst AbusTaylor \& Francis. 2019;40:102-15.

24. Price CJ, Hooven C. Interoceptive awareness skills for emotion regulation: theory and approach of mindful awareness in body-oriented therapy (MABT). Front Psychol. 2018;9:798.

25. Vidrine Jl, Spears CA, Heppner WL, Reitzel LR, Marcus MT, Cinciripini PM et al. Efficacy of mindfulness-based addiction treatment (MBAT) for smoking cessation and lapse recovery: a randomized clinical trial. J Consult Clin Psychol. 2016;84:824-38.

26. Spears CA, Hedeker D, Li L, Wu C, Anderson NK, Houchins SC, et al. Mechanisms underlying mindfulness-based addiction treatment versus cognitive behavioral therapy and usual care for smoking cessation. J Consult Clin Psychol. 2017:85:1029-40.

27. Brewer JA, Mallik S, Babuscio TA, Nich C, Johnson HE, Deleone CM, et al. Mindfulness training for smoking cessation: results from a randomized controlled trial. Drug Alcohol Depend. 2011;119:72-80.

28. Amaro H, Black DS. Moment-by-moment in Women's recovery: randomized controlled trial protocol to test the efficacy of a mindfulness-based intervention on treatment retention and relapse prevention among women in residential treatment for substance use disorder. Contemp Clin Trials. 2017;62:146-52.

29. Amaro H, Vallejo Z. Moment-by-moment in women's recovery: a mindfulness-based approach to relapse preventionAn Educ Gr Curric women Recover. Boston: Inst Urban Heal Res Northeast Univ Bost Public Heal Comm; 2009

30. Abed M, Ansari SM. Mindfulness-based relapse prevention to reduce lapse and craving. J Subst Use: Taylor \& Francis; 2019.

31. Brown DR, Jackson TCJ, Claus ED, Votaw VR, Stein ER, Robinson CSH, et al. Decreases in the late positive potential to alcohol images among alcohol treatment seekers following mindfulness-based relapse prevention. Alcohol Alcohol. 2020;55(1):78-85. https://doi.org/10.1093/alcalc/agz096.

32. Carroll H, Lustyk MKB. Mindfulness-based relapse prevention for substance use disorders: effects on cardiac vagal control and craving under stress. Mindfulness (N Y)Springer. 2018;9:488-99.

33. Davis JP, Berry D, Dumas TM, Ritter E, Smith DC, Menard C, et al. Substance use outcomes for mindfulness based relapse prevention are partially mediated by reductions in stress: results from a randomized trial. J Subst Abus TreatElsevier Science. 2018:91:37-48.

34. Davis JP, Barr N, Dworkin ER, Dumas TM, Berey B, DiGuiseppi G, et al. Effect of mindfulness-based relapse prevention on impulsivity trajectories among young adults in residential substance use disorder treatment. Mindfulness (N Y)Springer. 2019;10:1997-2009

35. Glasner-Edwards S, Mooney L, Ang A, Garneau HCHC, Hartwell E, Brecht M$\mathrm{L}$, et al. Mindfulness based relapse prevention for stimulant dependent adults: a pilot randomized clinical trial. Mindfulness (N Y)Springer. 2017;8: $126-35$

36. Hsiao Y-Y, Tofighi D, Kruger ES, Lee Van Horn M, MacKinnon DP, Witkiewitz $K$. The (lack of) replication of self-reported mindfulness as a mechanism of change in mindfulness-based relapse prevention for substance use disorders. Mindfulness (N Y)Springer. 2019;10:724-36.

37. Greenfield BL, Roos C, Hagler K, Stein E, Bowen S, Witkiewitz KA. Race/ ethnicity and racial group composition moderate the effectiveness of mindfulness-based relapse prevention for substance use disorder. Addict BehavElsevier Ltd. 2018;81:96-103.

38. Roos CR, Bowen $S$, Witkiewitz K. Baseline patterns of substance use disorder severity and depression and anxiety symptoms moderate the efficacy of mindfulness-based relapse prevention. J Consult Clin Psychol. 2017:85:1041-51.

39. Roos CR, Stein E, Bowen S, Witkiewitz K. Individual gender and group gender composition as predictors of differential benefit from mindfulness-based relapse prevention for substance use disorders. Mindfulness (N Y). 2019;10: 1560-67.

40. Shorey RC, Elmquist J, Gawrysiak MJ, Strauss C, Haynes E, Anderson S, et al. A randomized controlled trial of a mindfulness and acceptance group therapy for residential substance use patients. Subst Use MisuseTaylor \& Francis. 2017;52:1400-10.

41. Weiss de Souza IC, Kozasa EH, Bowen S, Richter KP, LMA S, Colugnati FAB, et al. Effectiveness of mindfulness-based relapse prevention program as an 
adjunct to the standard treatment for smoking: a pragmatic design pilot study. Nicotine Tob ResEngland. 2020;57(ntaa057). https://doi.org/10.1093/ ntr/ntaa057.

42. Witkiewitz K, Stein ER, Votaw VR, Wilson AD, Roos CR, Gallegos SJ, et al. Mindfulness-based relapse prevention and transcranial direct current stimulation to reduce heavy drinking: a double-blind sham-controlled randomized trial. Alcohol Clin Exp ResWiley-Blackwell Publishing Ltd. 2019; 43:1296-307.

43. Yaghubi M, Zargar F, Akbari H. Comparing effectiveness of mindfulnessbased relapse prevention with treatment as usual on impulsivity and relapse for methadone-treated patients: a randomized clinical trial. Addict Heal Iran. 2017;9:156-65.

44. Zemestani M, Ottaviani C. Effectiveness of mindfulness-based relapse prevention for co-occurring substance use and depression disorders. Mindfulness (N Y). Springer. 2016:7:1347-55.

45. Zgierska AE, Burzinski CA, Mundt MP, McClintock AS, Cox J, Coe CL, et al. Mindfulness-based relapse prevention for alcohol dependence: findings from a randomized controlled trial. J Subst Abus TreatElsevier. 2019;100:8-17.

46. Garland EL, Roberts-Lewis A, Tronnier CD, Graves R, Kelley K. Corrigendum to "mindfulness-oriented recovery enhancement versus CBT for cooccurring substance dependence, traumatic stress, and psychiatric disorders: proximal outcomes from a pragmatic randomized trial". Behav Res TherElsevier Science. 2018;100:78.

47. Garland EL, Baker AK, Howard MO. Mindfulness-oriented recovery enhancement reduces opioid attentional bias among prescription opioidtreated chronic pain patients. J Soc Soc Work ResUniv of Chicago Press. 2017:8:493-509.

48. Garland EL, Bryan CJ, Finan PH, Thomas EA, Priddy SE, Riquino MR, et al. Pain, hedonic regulation, and opioid misuse: modulation of momentary experience by mindfulness-oriented recovery enhancement in opioidtreated chronic pain patients. Drug Alcohol Depend. 2017;173:S65-72.

49. Garland EL, Howard MO, Zubieta J-K, Froeliger B. Restructuring hedonic dysregulation in chronic pain and prescription opioid misuse: effects of mindfulness-oriented recovery enhancement on responsiveness to drug cues and natural rewards. Psychother PsychosomNIH Public Access. 2017:86:111.

50. Garland EL, Hanley AW, Riquino MR, Reese SE, Baker AK, Salas K, et al. Mindfulness-oriented recovery enhancement reduces opioid misuse risk via analgesic and positive psychological mechanisms: a randomized controlled trial. J Consult Clin PsycholAmerican Psychological Association. 2019;87:92740.

51. Garland EL, Hanley AW, Kline A, Cooperman NA. Mindfulness-oriented recovery enhancement reduces opioid craving among individuals with opioid use disorder and chronic pain in medication assisted treatment: ecological momentary assessments from a stage 1 randomized controlled trial. Drug Alcohol Depend. 2019;203:61-5.

52. Garland EL, Atchley RM, Hanley AW, Zubieta J-K, Froeliger B. Mindfulnessoriented recovery enhancement remediates hedonic dysregulation in opioid users: neural and affective evidence of target engagement. Sci Adv. 2019:40:5.

53. Price CJ, Thompson EA, Crowell S, Pike K. Longitudinal effects of interoceptive awareness training through mindful awareness in bodyoriented therapy (MABT) as an adjunct to women's substance use disorder treatment: a randomized controlled trial. Drug Alcohol DependElsevier. 2019:198:140-9.

54. Somohano V, Shank T, Bowen S. Mindfulness-based relapse prevention for addictive behaviors. In: Handb mindfulness-based program. New York: Guilford Press; 2019. p. 341-53.

55. Bowen S, Chawla N, Collins SESE, Witkiewitz K, Hsu S, Grow J, et al. Mindfulness-based relapse prevention for substance use disorders: a pilot efficacy trial. Subst AbusTaylor \& Francis Haworth Press Plenum Publishing Corp. Springer. 2009;30:295-305.

56. Witkiewitz K, Warner K, Sully B, Barricks A, Stauffer C, Thompson BLBL, et al. Randomized trial comparing mindfulness-based relapse prevention with relapse prevention for women offenders at a residential addiction treatment center. Subst Use Misuse. 2014;49:536-46.

57. Zgierska A, Rabago D, Zuelsdorff M, Coe C, Miller M, Fleming M. Mindfulness meditation for alcohol relapse prevention: a feasibility pilot study. Addiction. 2008:2:165-73.

58. Imani S, Vahid MKA, Gharraee B, Noroozi A, Habibi M, Bowen S. Effectiveness of mindfulness-based group therapy compared to the usual opioid dependence treatment. Iran J PsychiatryTehran University of Medical Sciences. 2015;10:175.

59. Bowen S, Witkiewitz K, Clifasefi SL, Grow J, Chawla N, Hsu SHSH, et al. Relative efficacy of mindfulness-based relapse prevention, standard relapse prevention, and treatment as usual for substance use disorders: a randomized clinical trial. JAMA Psychiat. 2014;71:547-56.

60. Critchley HD, Garfinkel SN. Interoception and emotion. Curr Opin PsycholElsevier. 2017;17:7-14.

61. Michalak J, Mander J, Heidenreich T. Implementation and dissemination of mindfulness-based interventions. Mindfulness (N Y)Springer. 2020;11:275-8.

62. Herman PM, Anderson ML, Sherman KJ, Balderson BH, Turner JA, Cherkin DC. Cost-effectiveness of mindfulness-based stress reduction versus cognitive behavioral therapy or usual care among adults with chronic low Back pain. Spine (Phila Pa 1976)Lippincott Williams and Wilkins. 2017;42: 1511-20.

\section{Publisher's Note}

Springer Nature remains neutral with regard to jurisdictional claims in published maps and institutional affiliations.

Ready to submit your research? Choose BMC and benefit from:

- fast, convenient online submission

- thorough peer review by experienced researchers in your field

- rapid publication on acceptance

- support for research data, including large and complex data types

- gold Open Access which fosters wider collaboration and increased citations

- maximum visibility for your research: over $100 \mathrm{M}$ website views per year

At BMC, research is always in progress.

Learn more biomedcentral.com/submissions 